TRANSACTIONS OF THE AMERICAN MATHEMATICAL SOCIETY

Volume 348, Number 9, September 1996

\title{
SEIFERT MANIFOLDS WITH FIBER SPHERICAL SPACE FORMS
}

\author{
JONG BUM LEE, KYUNG BAI LEE, AND FRANK RAYMOND
}

\begin{abstract}
We study the Seifert fiber spaces modeled on the product space $S^{3} \times \mathbb{R}^{2}$. Such spaces are "fiber bundles" with singularities. The regular fibers are spherical space-forms of $S^{3}$, while singular fibers are finite quotients of regular fibers. For each of possible space-form groups $\Delta$ of $S^{3}$, we obtain a criterion for a group extension $\Pi$ of $\Delta$ to act on $S^{3} \times \mathbb{R}^{2}$ as weakly $S^{3}$ equivariant maps, which gives rise to a Seifert fiber space modeled on $S^{3} \times \mathbb{R}^{2}$ with weakly $S^{3}$-equivariant maps $\operatorname{TOP}_{S^{3}}\left(S^{3} \times \mathbb{R}^{2}\right)$ as the universal group. In the course of proving our main results, we also obtain an explicit formula for $H^{2}(Q ; \mathbb{Z})$ for a cocompact crystallographic or Fuchsian group $Q$. Most of our methods for $S^{3}$ apply to compact Lie groups with discrete center, and we state some of our results in this general context.
\end{abstract}

\section{INTRODUCTION}

There have been many successful investigations of fiberings with singularities. We have been concerned with fiberings where the typical fiber is the quotient $\Gamma \backslash G$ of a Lie group $G$ by a cocompact lattice $\Gamma \subset G$. The singular fibers are then quotients of $\Gamma \backslash G$ by finite groups of affine diffeomorphisms. A viable theory with $G$ connected abelian was developed by Conner and Raymond in several works [2], [3], [4]. This was carried further in a series of papers by the current authors and expanded to include nilpotent and certain solvable $G$. The fiber structure is useful and often crucial for applications such as determining what groups can act effectively on flat or almost flat manifolds. See [4], [9] and [8] for a careful exposition and many other geometric applications.

In a short paper [10], we introduced the notion of Seifert fiberings modeled on principal $G$-bundles. Here $G$ was an arbitrary Lie group and the theory was set up so that it included all the previous work. However, nothing really explicit involving concrete calculations for groups other than solvable groups are in the literature, except possibly [17].

In this paper, we shall examine Seifert fiberings modeled on product $G$-bundles, $G \times W$, where $G$ is the simple group $S^{3}$. Here the typical fibers are 3-dimensional

Received by the editors December 1, 1994 and, in revised form, September 7, 1995.

1991 Mathematics Subject Classification. Primary 57M50; Secondary 55R60, 57M05, 57M60.

Key words and phrases. Seifert fiber space, spherical space-form, Fuchsian group, crystallographic group, group extension, cohomology of groups.

The first author was supported in part by the Basic Science Research Institute Program, Ministry of Education, 1994, Project No. BSRI-94-1422, and by TGRC-KOSEF, Korea.

The third author was supported in part by National Science Foundation grant DMS-9306240, U.S.A.

(C)1996 American Mathematical Society 
spherical space-forms. Even more concretely, when we require $W$ to be homeomorphic to $\mathbb{R}^{2}$, the Seifert fiberings become explicitly determined closed 5-dimensional manifolds over 2-dimensional orbifolds.

From our point of view, the classical 3-dimensional Seifert manifold $M$ consists of a "model space" $P$ and a "universal group" Isom $(P)$. For example, we may choose $P=\widetilde{\operatorname{PSL}(2, \mathbb{R}})$, the universal covering of $\operatorname{PSL}(2, \mathbb{R})$ and, $\operatorname{Isom}(P)=(\mathbb{R} \times \mathbb{Z}$ $\widehat{\operatorname{PSL}(2, \mathbb{R})}) \rtimes \mathbb{Z}_{2}$. Note, in general, $P$ is a principal $G$-fibering with fiber either $G=\mathbb{R}$ or $S^{1}$, and $\operatorname{Isom}(P)$ is weakly $G$-equivariant. A Seifert manifold is then $M=\Pi \backslash P$, with $\Pi$ discrete in $\operatorname{Isom}(P)$ and acting properly (automatically) and freely on $P$. The manifold $M$ inherits the geometry from $P$ when $\Pi$ acts freely. If $\Pi$ does not act freely, we obtain a Seifert orbifold. (Observe that classical Seifert 3-manifolds over bad 2-dimensional orbifolds can be regarded as Seifert orbifolds coming from $\left(S^{3}, \operatorname{Isom}\left(S^{3}\right)\right)$.)

In general, the model space $P$ will be a principal $G$-bundle over a space $W$ with a general Lie group $G$. For the universal group, we use $\operatorname{TOP}_{G}(P)$, the group of weakly $G$-equivariant homeomorphisms of $P$. Sometimes we use smaller subgroups, like $\operatorname{Isom}_{G}(P)=\operatorname{Isom}(P) \cap \mathrm{TOP}_{G}(P)$, to get stronger geometries as in the classical case mentioned above. A discrete group $\Pi \subset \operatorname{TOP}_{G}(P)$ acting on $P$ properly, yields a Seifert fiber space $M=\Pi \backslash P$. Although it is not necessary, we shall require that $\Gamma=\Pi \cap \ell(G)$ be a cocompact lattice of $G$, where $\ell(G)$ is the principal left $G$-action. The space $M$ inherits a "fiber structure" coming from the principal fibering $G \rightarrow P \rightarrow W$. If $\Pi$ acts freely, then $P \rightarrow M$ is an unbranched covering. Otherwise, it will be branched. Since the group $\Pi$ normalizes the left $G$-action on $P$, we obtain the group $G \cdot \Pi$ acting on $P$ with the fibering $\Pi \backslash P=M \rightarrow B=$ $(G \cdot \Pi) \backslash P=(\Pi / \Pi \cap G) \backslash(G \backslash P)=Q \backslash W$, where $Q=\Pi /(\Pi \cap G)$. That is, we have the following diagram of maps coming from the proper actions of $G \cdot \Pi$ on $P$ :

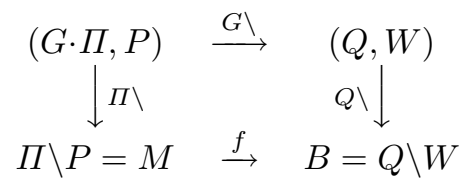

The bottom map is the singular fibering. Now each "fiber" $f^{-1}(b)$ can be described by examining the action of $G \cdot \Pi$ on $(G \cdot \Pi)(u)$, where $u$ is on the $G \cdot \Pi$ orbit of $b \in B$. In this context, $f^{-1}(b)$ is $\Gamma \backslash G$ with $\Gamma=G \cap \Pi$, or a finite quotient of this homogeneous space where the finite group is isomorphic to the stabilizer $Q_{w}$ of the $Q$ orbit $G \backslash(G \cdot \Pi)(u)=w \in W$. Furthermore, this group $Q_{w}$ acts, in a controlled way, as diffeomorphisms on $\Gamma \backslash G$ induced from $G \rtimes \operatorname{Aut}(G)$.

An explicit description is as follows: Let $\Gamma \subset \ell(G)$ be a lattice, and $\rho: Q \rightarrow$ $\operatorname{TOP}(W)$ be a properly discontinuous action. Consider an extension of $\Gamma$ by $Q$,

$$
1 \rightarrow \Gamma \rightarrow \Pi \rightarrow Q \rightarrow 1
$$

Thus $\operatorname{TOP}_{G}(P)$, the topological group of homeomorphisms which normalize the left $G$-action on $P$, is our universal group and $P$ is our model space. We want in this paper to characterize those $\Pi$ which act on the product $P=G \times W$ as weakly $G$ equivariant homeomorphisms. The group $\Pi$ must map into $\operatorname{TOP}_{G}(P)$, and $\Pi \cap G=$ $\Gamma$. This is the same as studying representations $\theta$ of $\Pi$ into $\operatorname{TOP}_{G}(G \times W)$ which extend $\Gamma \subset \ell(G)$ and inducing $\rho: Q \rightarrow \operatorname{TOP}(W)$. The action of $\Pi$ on $G \times W$ will 
be properly discontinuous, and it will yield a Seifert fiber space

$$
\Gamma \backslash G \rightarrow \Pi \backslash(G \times W) \rightarrow Q \backslash W
$$

with typical fiber $\Gamma \backslash G$ and base orbifold $Q \backslash W$. Each such homomorphism $\theta: \Pi \rightarrow$ $\mathrm{TOP}_{G}(P)$ is called a Seifert Construction.

Reducing the universal group $\mathrm{TOP}_{G}(G \times W)$ to a subgroup that still contains all the left translations has the effect of imposing additional structure on the Seifert fiberings $\Pi \backslash(G \times W)$. For example, if $G$ has a left-invariant metric and $W$ is a Riemannian manifold, we may impose the product metric on $G \times W$ and require that $\Pi \hookrightarrow \mathrm{TOP}_{G}(G \times W) \cap \operatorname{Isom}(G \times W)$. Then, if $\Pi$ acts freely on $G \times W$, $M=\Pi \backslash(G \times W)$ would inherit a product geometry from $G \times W$, and the fibers would be Riemannian submanifolds of $M$.

When $G=\mathbb{R}^{n}$, simply connected nilpotent or solvable of type (R) (i.e., all eigenvalues of $\operatorname{ad}(g)$ are real for every $g \in G)$, every extension gives rise to a Seifert construction. Furthermore, after fixing $\phi \times \rho: Q \rightarrow \operatorname{Out}(G) \times \operatorname{TOP}(W)$, each embedding is unique up to conjugation by elements of the universal group $\mathrm{TOP}_{G}(P)$. This is due largely to two facts about these groups $G$ :

1. Lattices $\Gamma$ in $G$ have the unique automorphism extension property.

2. The cohomology groups $H^{i}(Q)$ with certain coefficients vanish for $i=1,2$.

This state of affairs does not hold for general $G$ nor for our special case of $G=$ $S^{3}$. Extensions may not embed and, if they do, they are not necessarily unique. The group $G=S^{3}$ is a good choice to begin to understand the complexities and complications of the general case, because $S^{3}$ is a compact, simply connected, simple non-trivial Lie group of least dimension. Moreover, it is especially relevant since it ties in very nicely with 3-dimensional geometry and topology.

In the second section, we define precisely and characterize the universal group for a product $G$-bundle $P=G \times W$. For our exploration, we additionally require that $\operatorname{Aut}(G)$ splits as $\operatorname{Inn}(G) \rtimes \operatorname{Out}(G)$, which is automatically true for $G=S^{3}$. The Main Lemma 2.2 then determines what we must do to solve the embedding problem of our extension into $\mathrm{TOP}_{G}(G \times W)$ (i.e., finding a Seifert construction). Theorem 3.6 then tells us what the criteria are to embed $\Pi$ when $G=S^{3}$ for general $(Q, W)$ and $\Delta$, a 3-dimensional spherical space-form group lying in $G \times G$ which replaces the less general lattice $\Gamma \subset G$. Section 4 then carries out the program for $(Q, W)$, where $Q$ is a cocompact group of orientation-preserving proper homeomorphisms of $\mathbb{R}^{2}$. This is equivalent to assuming that $Q$ is a crystallographic or Fuchsian group acting topologically on the euclidean or hyperbolic plane. Under these conditions we obtain, in Theorem 4.7, a remarkably clear answer to the embedding problem. We can easily discern those that possess 5 -dimensional geometries which are the product of spherical 3-dimensional geometry and hyperbolic or euclidean plane geometry. In the course of establishing 4.7, in Theorem 4.5 (and the Remark following it) we obtain an explicit formula for $H^{*}(Q ; \mathbb{Z})$, and an integer invariant for each mod $n$ reduction class of elements of $H^{2}(Q ; \mathbb{Z})$. In Theorem 5.4, we obtain a complete solution for deformation of Seifert fiber structures in terms of group cohomology. An example is worked out in 4.8 and 5.5.

\section{Structure of the Universal Group}

Let $G$ be a Lie group and $W$ be a locally compact Hausdorff space which admits covering space theory. We view the product $G \times W$ as a principal $G$-bundle. 
We denote by $\mathrm{M}(W, G)$ the group of all continuous maps from $W$ into $G$ with multiplication

$$
(\lambda \cdot \nu)(w)=\lambda(w) \cdot \nu(w)
$$

$\operatorname{TOP}(W)$ denotes the topological group of all homeomorphisms of $W$, and $\operatorname{Aut}(G)$ denotes the Lie group of all continuous automorphisms of $G$. Then $\operatorname{Aut}(G) \times$ $\operatorname{TOP}(W)$ acts on $\mathrm{M}(W, G)$ via

$$
{ }^{(\alpha, h)} \lambda=\alpha \circ \lambda \circ h^{-1}
$$

for $(\alpha, h) \in \operatorname{Aut}(G) \times \operatorname{TOP}(W)$ and $\lambda \in \mathrm{M}(W, G)$. The group $\operatorname{TOP}_{G}(G \times W)$ of weakly $G$-equivariant self-homeomorphisms of $G \times W$ is defined as follows: A homeomorphism $f$ of $G \times W$ onto itself belongs to $\operatorname{TOP}_{G}(G \times W)$ if and only if there exists a continuous automorphism $\alpha_{f}$ of $G$ so that

$$
f(a \cdot x, w)=\alpha_{f}(a) f(x, w)
$$

for all $a \in G$ and $(x, w) \in G \times W$.

It is known [9] that

$$
\mathrm{TOP}_{G}(G \times W)=\mathrm{M}(W, G) \rtimes(\operatorname{Aut}(G) \times \operatorname{TOP}(W)) .
$$

The group law is

$$
\left(\lambda_{1}, \alpha_{1}, h_{1}\right) \cdot\left(\lambda_{2}, \alpha_{2}, h_{2}\right)=\left(\lambda_{1} \cdot \alpha_{1} \circ \lambda_{2} \circ h_{1}^{-1}, \alpha_{1} \circ \alpha_{2}, h_{1} \circ h_{2}\right),
$$

and the action of $\operatorname{TOP}_{G}(G \times W)$ on $G \times W$ is given by

$$
(\lambda, \alpha, h) \cdot(x, w)=\left(\alpha(x) \cdot \lambda(h(w))^{-1}, h(w)\right) .
$$

Then $\mathrm{M}(W, G) \rtimes \operatorname{Aut}(G)$ is the group of all weakly $G$-equivariant self-homeomorphisms of $G \times W$ which move only along the fibers. (In the past, the action of $\lambda$ that was used was multiplication by $\lambda(w)$ rather than $\lambda(w)^{-1}$, which caused the group law of $\mathrm{M}(W, G)$ to be written in a reversed order. Here we chose this more convenient form.)

For $a \in G$, the constant map $W \rightarrow G$ sending $W$ to $a$ is denoted by

$$
r(a)=(a, 1) \in \mathrm{M}(W, G) \rtimes \operatorname{Aut}(G) .
$$

This is a right translation by $a^{-1}$ on the first factor of $G \times W$ so that $r(a)(x, w)=$ $\left(x \cdot a^{-1}, w\right)$, and the subgroup of all such right translations is denoted by $r(G) \subset$ $\mathrm{M}(W, G)$. Let $\ell(G)$ denote the group of left translations on the first factor, so that $\ell(a)(x, w)=(a \cdot x, w)$. Then elements of $\ell(G)$ are of the form

$$
\ell(a)=\left(a^{-1}, \mu(a)\right) \in \mathrm{M}(W, G) \rtimes \operatorname{Inn}(G),
$$

where $\mu(a)$ is conjugation by $a \in G$ so that $\mu(a)(x)=a x a^{-1}$. Note that $\ell(G)$ is normal in $\mathrm{TOP}_{G}(G \times W)$. In fact, our $\operatorname{TOP}_{G}(G \times W)$ is the largest subgroup of $\operatorname{TOP}(G \times W)$ in which $\ell(G)$ is normal. We call the group $\operatorname{TOP}_{G}(G \times W)$ the universal group for the Seifert construction. Recall that it is the group of all weakly $G$-equivariant self-homeomorphisms of $G \times W$.

Let $G$ be a Lie group such that $\operatorname{Aut}(G)=\operatorname{Inn}(G) \rtimes \operatorname{Out}(G)$. For example, $\mathbb{R}^{n}$ or a semi-simple Lie group of non-compact type with trivial center satisfies this condition. From now on, we use a specific fixed lifting of $\operatorname{Out}(G)$ into $\operatorname{Aut}(G)$. 
Then every $\alpha \in \operatorname{Aut}(G)$ is written uniquely as $\mu(a) \circ \bar{\alpha}$, for some $a \in G$ and $\bar{\alpha} \in \operatorname{Out}(G) \subset \operatorname{Aut}(G)$. We work with

$$
\begin{aligned}
\mathcal{U} & =\mathrm{M}(W, G) \rtimes(\operatorname{Aut}(G) \times \operatorname{TOP}(W)) \\
& =\mathrm{M}(W, G) \rtimes((\operatorname{Inn}(G) \rtimes \operatorname{Out}(G)) \times \operatorname{TOP}(W))
\end{aligned}
$$

as the universal group for a Seifert fiber space construction for $G$.

2.1. Lemma. Let $G$ be a Lie group such that $\operatorname{Aut}(G)=\operatorname{Inn}(G) \rtimes \operatorname{Out}(G)$. Then $\mathcal{U}$ can be written as

$$
\mathcal{U}=\ell(G) \rtimes_{\mathcal{Z}(G)}[\mathrm{M}(W, G) \rtimes(\operatorname{Out}(G) \times \operatorname{TOP}(W))] .
$$

Proof. If $\alpha=\mu(a) \circ \bar{\alpha}$, we can write $(\lambda, \alpha, h)$ as

$$
\begin{aligned}
(\lambda, \alpha, h) & =(\lambda, \mu(a) \circ \bar{\alpha}, h) \\
& =\left(a^{-1}\left(a(\lambda a) a^{-1}\right), \mu(a) \circ \bar{\alpha}, h\right) \\
& =\left(a^{-1}, \mu(a), 1,\right) \cdot(\lambda a, \bar{\alpha}, h) .
\end{aligned}
$$

This shows $\ell(G)$ and $\mathrm{M}(W, G) \rtimes(\operatorname{Out}(G) \times \operatorname{TOP}(W))$ generate $\mathrm{M}(W, G) \rtimes(\operatorname{Aut}(G) \times$ $\operatorname{TOP}(W))$. Clearly, the center $\mathcal{Z}(G)$ of $G$ lies in $\ell(G)$ and $r(G) \subset \mathrm{M}(W, G)$, and $\ell(a)=\left(a^{-1}, \mu(a), 1\right)$ and $r\left(a^{-1}\right)=\left(a^{-1}, 1,1\right)$ for $a \in \mathcal{Z}(G)$ define the same elements of $\mathcal{U}$.

Define $\tilde{\mathcal{U}}$ and $\overline{\mathcal{U}}$ by

$$
\begin{aligned}
& \tilde{\mathcal{U}}=\ell(G) \rtimes[\mathrm{M}(W, G) \rtimes(\operatorname{Out}(G) \times \operatorname{TOP}(W))], \\
& \overline{\mathcal{U}}=\mathrm{M}(W, G) \rtimes(\operatorname{Out}(G) \times \operatorname{TOP}(W)) .
\end{aligned}
$$

Then, by Lemma 2.1,

$$
\mathcal{U}=\ell(G) \rtimes_{\mathcal{Z}(G)} \overline{\mathcal{U}}
$$

Our chief concern in this paper is for $G=S^{3}$. We note then that $\operatorname{Out}(G)$ is trivial, and consequently we have

$$
\begin{aligned}
& \widetilde{\mathcal{U}}=\ell(G) \times[\mathrm{M}(W, G) \rtimes \operatorname{TOP}(W)], \\
& \overline{\mathcal{U}}=\mathrm{M}(W, G) \rtimes \operatorname{TOP}(W), \\
& \mathcal{U}=\ell(G) \rtimes_{\mathbb{Z}_{2}} \overline{\mathcal{U}} .
\end{aligned}
$$

Now suppose $\Gamma \subset \ell(G)$ is a lattice, and $\rho: Q \rightarrow \operatorname{TOP}(W)$ is an action which is not necessarily effective. With an extension $1 \rightarrow \Gamma \rightarrow \Pi \rightarrow Q \rightarrow 1$, our goal is finding a homomorphism $\theta: \Pi \rightarrow \mathcal{U}$ which gives rise to an action of $\Pi$ on $G \times W$. For $\theta$ to be injective, it is necessary that the kernel of $\phi \times \rho: Q \rightarrow \operatorname{Out}(G) \times \operatorname{TOP}(W)$ be mapped injectively to $\ell(G) \times_{\mathcal{Z}(G)} \mathrm{M}(W, G)$. Let $\Delta=\theta(\Pi) \cap[\mathrm{M}(W, G) \rtimes \operatorname{Inn}(G)]$. In the case when $G$ is simply connected nilpotent, or more generally, solvable of type (R) [8], for $\Delta$ to act properly on $G$, it must be a lattice of $G$ again, and lie inside $\ell(G) \subset r(G) \rtimes \operatorname{Inn}(G)=\ell(G) \times_{\mathcal{Z}(G)} r(G)$. This can be seen as follows: Suppose $\Delta \subset \mathrm{M}(W, G) \rtimes \operatorname{Inn}(G)$ acts properly on $G \times W$. Since it acts trivially on the $W$-factor, it acts on $G$ properly. Therefore, $Q^{\prime}=\Delta / \Gamma$ must be finite, and injects into $\mathrm{M}(W, G) / \mathcal{Z}(G)$ by the natural projection $\mathrm{M}(W, G) \rtimes \operatorname{Inn}(G)=$ $\ell(G) \rtimes_{\mathcal{Z}(G)} \mathrm{M}(W, G) \rightarrow \mathrm{M}(W, G) / \mathcal{Z}(G)$. However, for all Lie groups listed above, $\mathrm{M}(W, G) / \mathcal{Z}(G)$ does not have elements of finite order except the identity. This is because the exponential map $\mathfrak{g} \rightarrow G$ is bijective. Thus the equation $x^{n}=a$ on $G$ 
has a unique solution for every $n \in \mathbb{Z}$ and every $a \in G$. This shows that $\Delta \subset G$. For $G=S^{3}$, this is not true any more. In fact, an abstract lattice $\Gamma$ can sit in $\mathrm{M}(W, G) \rtimes \operatorname{Inn}(G)$ with $\Gamma \cap \ell(G)$ even trivial. To control such situations, we shall use $\Delta \subset \ell(G) \times \mathcal{Z}(G) r(G)$ instead of $\Gamma$, where $r(G)$ is the group of right translations on the first factor of $G \times W$. In fact, to avoid complication, we shall use subgroups $\Delta^{*}$ of $\ell(G) \times r(G)$ rather than of $\ell(G) \times \mathcal{Z}(G) r(G)$. Note that some finite subgroups $\Delta \subset \ell(G) \times \times_{\mathcal{Z}(G)} r(G)$ lift to $\ell(G) \times r(G)$ and some do not. For the latter case, $\Delta^{*}$ will then be the covering group of $\Delta$, so that $\Delta=\Delta^{*} / \mathcal{Z}(G)$, where $\mathcal{Z}(G)$ is the center of $G$ sitting diagonally. If we use $\Delta \subset \ell(G) \times \mathcal{Z}(G) r(G)$, and replace the exact sequence $1 \rightarrow \Gamma \rightarrow \Pi \rightarrow Q \rightarrow 1$ by $1 \rightarrow \Delta \rightarrow \Pi \rightarrow Q^{\prime} \rightarrow 1$, where $Q^{\prime}=\Pi / \Delta=Q /(\Delta / \Gamma)$, nothing is lost in generality, but more geometry is put into our embedding problem when $G$ is compact. (If $G$ is $\mathbb{R}^{n}$ or a simply connected nilpotent Lie group or solvable Lie group of type $(\mathrm{R})$, nothing would be gained by this move.)

Suppose $W=\mathbb{R}^{2}$ or $\mathbf{H}$, and $Q$ is either a euclidean crystallographic or Fuchsian group, respectively. Then the typical fiber of the resulting Seifert fiber space is $(\Delta \cap \ell(G)) \backslash G$, and the base is a 2-dimensional compact Riemannian orbifold. Actually there will be no "typical fiber" unless $\Delta$ lies purely in $\ell(G)$. This is due to the way the term typical fiber is defined. What we actually see over all the non-branched points is $\Delta \backslash S^{3}$. Over a branched point $w \in Q \backslash W$ with multiplicity $\alpha_{j}$, we find that the fiber is the orbit space of $\Delta \backslash S^{3}$ by a cyclic group $\mathbb{Z}_{\alpha_{j}}$ acting, up to affine equivalence, as isometries.

Since we require that $\Delta$ maps into $\ell(G) \times_{\mathcal{Z}(G)} r(G), \phi \times \rho$ will be injective if $\theta$ is to be injective.

Dividing out by $\mathcal{Z}(G)$ causes a lot of unnecessary trouble. Therefore, to avoid such trouble, we use $\widetilde{\mathcal{U}}$ instead of $\mathcal{U}$ itself. Hence we work with $\Delta^{*} \subset \ell(G) \times r(G)$ rather than $\Delta \subset \ell(G) \times \mathcal{Z}(G) r(G)$. This procedure is useful when $\mathcal{Z}(G)$ is small, say a finite group. For $G=S^{3}$, it is $\mathbb{Z}_{2}$. We shall use the same notation for subgroups $\Delta \subset \ell(G) \times \mathcal{Z}(G) r(G)$ and $\Delta^{*} \subset \ell(G) \times r(G)$, unless we need to distinguish them clearly. It should be clear from the context. Therefore, $\Delta$ may mean a subgroup of $\mathcal{U}$ or $\widetilde{\mathcal{U}}$.

The following lemma says that making a Seifert construction $\Pi \rightarrow \widetilde{\mathcal{U}}$ is equivalent to constructing a homomorphism of $\Pi$ extending the homomorphism of $\Delta$. This breaks the construction problem into two pieces, each of which can be addressed separately.

2.2. Main Lemma. Let $G$ be a Lie group such that $\operatorname{Aut}(G)=\operatorname{Inn}(G) \rtimes \operatorname{Out}(G)$. Suppose $1 \rightarrow \Delta \rightarrow \Pi \rightarrow Q \rightarrow 1$ is exact, $i=i_{\ell} \times i_{r}: \Delta \hookrightarrow \ell(G) \times r(G)$, and $\rho: Q \hookrightarrow \operatorname{TOP}(W)$ is an action. In order for there to exist a homomorphism $\theta: \Pi \rightarrow \widetilde{\mathcal{U}}$ which makes the diagram

$$
\begin{aligned}
& \begin{array}{lllllll}
1 & \rightarrow & \Delta & \rightarrow & \Pi & \rightarrow & Q
\end{array} \\
& \downarrow \quad \downarrow \theta \quad \downarrow \phi \times \rho \\
& 1 \rightarrow \ell(G) \times \mathrm{M}(W, G) \rightarrow \tilde{\mathcal{U}} \rightarrow \operatorname{Out}(G) \times \mathrm{TOP}(W) \rightarrow 1
\end{aligned}
$$

commutative, the following conditions are necessary and sufficient:

(a) The abstract kernel $Q \rightarrow \operatorname{Out}(\Delta)$ induces a homomorphism $\phi: Q \rightarrow \operatorname{Out}(G)$.

(b) There exists a homomorphism $\Pi \rightarrow \ell(G) \rtimes \operatorname{Out}(G)$ extending $i_{\ell}: \Delta \rightarrow \ell(G)$, and compatible with $\phi$. 
(c) There exists a homomorphism $\Pi \rightarrow \overline{\mathcal{U}}=\mathrm{M}(W, G) \rtimes(\operatorname{Out}(G) \times \operatorname{TOP}(W))$ extending $i_{r}: \Delta \rightarrow r(G)$, and compatible with $\phi$.

Proof. (Necessity) Suppose there exists such a homomorphism $\theta$. Observe that the subgroups of $\tilde{\mathcal{U}}, \mathrm{M}(W, G) \rtimes \operatorname{TOP}(W), \ell(G) \times[\mathrm{M}(W, G) \rtimes \operatorname{TOP}(W)], \mathrm{M}(W, G) \rtimes$ $(\operatorname{Out}(G) \times \operatorname{TOP}(W))$, and $\ell(G)$ are normal in $\widetilde{\mathcal{U}}$. Thus, $\theta$ followed by the appropriate quotient map by one of the above normal subgroups is a desired homomorphism.

(Sufficiency) The factor $\mathrm{M}(W, G) \rtimes \operatorname{TOP}(W)$ of $\overline{\mathcal{U}}$ commutes with $\ell(G)$, and only the $\operatorname{Out}(G)$-factor acts non-trivially on $\ell(G)$. Thus, the action of $\overline{\mathcal{U}}$ on $\ell(G)$ is only through $\operatorname{Out}(G)$. Since the homomorphisms $\Pi \rightarrow \ell(G) \rtimes \operatorname{Out}(G)$ and $\Pi \rightarrow \overline{\mathcal{U}}$ share the same $\Pi \rightarrow \operatorname{Out}(G)$, they yield a desired homomorphism $\theta: \Pi \rightarrow \widetilde{\mathcal{U}}$.

This lemma enables us to divide the embedding problem into 2 steps. Let $K_{\ell}$ be the kernel of $i_{\ell}: \Delta \rightarrow \ell(G)$. Then, as we shall see in Theorem 3.2, $K_{\ell}$ and $K_{r}$ are characteristic in $\Delta$, and hence are normal in $\Pi$. We map $\Pi / K_{\ell}$ into $\ell(G) \rtimes$ Out $(G)$ extending $\Delta \rightarrow \ell(G)$. Now let $K_{r}$ be the kernel of $i_{r}: \Delta \rightarrow r(G)$. As before, we map $\Pi / K_{r}$ into $\overline{\mathcal{U}}$, using the extension $1 \rightarrow i_{r}(\Delta) \rightarrow \Pi / K_{r} \rightarrow Q \rightarrow 1$ together with $\phi \times \rho: Q \rightarrow \operatorname{Out}(G) \times \operatorname{TOP}(W)$. Then we combine these two homomorphisms to construct a desired homomorphism $\Pi \rightarrow \widetilde{\mathcal{U}}$. We may express the desired homomorphism as the "composition" $\Pi \rightarrow \Pi \times \Pi \rightarrow \Pi / K_{\ell} \times \Pi / K_{r} \rightarrow$ $(\ell(G) \rtimes \operatorname{Out}(G)) \times(\mathrm{M}(W, G) \rtimes(\operatorname{Out}(G) \times \operatorname{TOP}(W))) \rightarrow \ell(G) \rtimes[\mathrm{M}(W, G) \rtimes \operatorname{Out}(G)] \rtimes$ $\operatorname{TOP}(W)$, where the first homomorphism is the diagonal map. Observe that it does not matter which homomorphism (b) or (c) in the statement is constructed first.

\section{Seifert Fiberings with $G=S^{3}$}

Now we specialize to the case where $G=S^{3}$. Then it has center $\mathcal{Z}(G)=\mathbb{Z}_{2}$, and $\operatorname{Aut}(G)=\operatorname{Inn}(G)$ since $\operatorname{Out}(G)$ is trivial. Therefore,

$$
\begin{aligned}
& \tilde{\mathcal{U}}=\ell(G) \rtimes[\mathrm{M}(W, G) \rtimes \operatorname{TOP}(W)]=[\ell(G) \times \mathrm{M}(W, G)] \rtimes \operatorname{TOP}(W), \\
& \overline{\mathcal{U}}=\mathrm{M}(W, G) \rtimes \operatorname{TOP}(W), \\
& \mathcal{U}=\ell(G) \rtimes_{\mathbb{Z}_{2}} \overline{\mathcal{U}} .
\end{aligned}
$$

Here is the list of all the finite subgroups of $S^{3}$ :

cyclic group

binary dihedral group

binary tetrahedral group

binary octahedral group

binary icosahedral group

$$
\begin{aligned}
\mathbb{Z}_{k} & =\left\langle x \mid x^{k}=1\right\rangle, \\
\mathbf{D}_{4 k}^{*} & =\left\langle x, y \mid x^{2 k}=1, x^{k}=y^{2}, y x y^{-1}=x^{-1}\right\rangle, \\
\mathbf{T}^{*} & =\left\langle x, y \mid x^{4}=1, x^{2}=(x y)^{3}=y^{3}\right\rangle, \\
\mathbf{O}^{*} & =\left\langle x, y \mid x^{4}=1, x^{2}=(x y)^{3}=y^{4}\right\rangle, \\
\mathbf{I}^{*} & =\langle x, y| x^{4}=1, x^{2}=(x y)^{3}=y^{5}
\end{aligned}
$$

Note that $\mathbf{D}_{4 k}^{*}=\mathbb{Z}_{2 k} \rtimes_{\mathbb{Z}_{2}} \mathbb{Z}_{4}$. When $k$ is odd, then $\mathbf{D}_{4 k}^{*}$ is also isomorphic to $\mathbb{Z}_{k} \rtimes \mathbb{Z}_{4}$. In fact,

$$
\mathbb{Z}_{k} \rtimes \mathbb{Z}_{4}=\left\langle x^{\prime}, y \mid{x^{\prime}}^{k}=1, y^{4}=1, y x^{\prime} y^{-1}=x^{\prime-1}\right\rangle,
$$

where $x^{\prime}=x^{2}$. It is enough to see that $x \in \mathbb{Z}_{k} \rtimes \mathbb{Z}_{4}$. Let $k=2 m+1$. Then, $y^{2}=x^{k}=x^{2 m+1}=x^{\prime m} \cdot x$. Thus, $x=x^{\prime-m} \cdot y^{2} \in \mathbb{Z}_{k} \rtimes \mathbb{Z}_{4}$. 
It is convenient to look at $G=S^{3}$ as a subgroup of $\mathrm{SO}(4)$. We may identify $G$ with the subgroup of $\mathrm{SO}(4)$ consisting of elements of the form

$$
\left[\begin{array}{rrrr}
x_{0} & -x_{1} & -x_{2} & -x_{3} \\
x_{1} & x_{0} & -x_{3} & x_{2} \\
x_{2} & x_{3} & x_{0} & -x_{1} \\
x_{3} & -x_{2} & x_{1} & x_{0}
\end{array}\right]
$$

with $x_{0}^{2}+x_{1}^{2}+x_{2}^{2}+x_{3}^{3}=1$. This corresponds to a unit quaternion $\mathbf{x}=x_{0}+x_{1} \mathbf{i}+$ $x_{1} \mathbf{j}+x_{1} \mathbf{k}$. So, if we identify the first column as $\mathbf{x}$, then the second, third and fourth columns are $\mathbf{x i}, \mathbf{x} \mathbf{j}$, and $\mathbf{x k}$, respectively. Let

$$
\sigma=\left[\begin{array}{rrrr}
-1 & 0 & 0 & 0 \\
0 & 1 & 0 & 0 \\
0 & 0 & 1 & 0 \\
0 & 0 & 0 & 1
\end{array}\right] .
$$

Conjugation by $\sigma$ is an involution of $\mathrm{SO}(4)$, changing the signs of the first row and the first column (therefore, the $(1,1)$-entry does not change). We denote the image $\sigma(G)$ by $G^{\sigma}$. Clearly $-I \in G \cap G^{\sigma}=\mathbb{Z}_{2}$, which is the center of $G$ and $G^{\sigma}$, and also of $\mathrm{SO}(4)$. Thus, $G \cdot G^{\sigma}=\mathrm{SO}(4)$ and $G \cap G^{\sigma}=\mathcal{Z}(\mathrm{SO}(4)) \cong \mathbb{Z}_{2}$, the center of $\mathrm{SO}(4)$. Therefore, every element of $\mathrm{SO}(4)$ can be written as $a \cdot b^{\sigma}=(-a) \cdot(-b)^{\sigma}$, with $a, b \in G$, uniquely modulo $\mathcal{Z}(\mathrm{SO}(4))$.

In the following, $R(\theta)$ denotes the rotation

$$
R(\theta)=\left[\begin{array}{rr}
\cos 2 \pi \theta & -\sin 2 \pi \theta \\
\sin 2 \pi \theta & \cos 2 \pi \theta
\end{array}\right]
$$

Note that

$$
\mathcal{R}(\theta)=\left[\begin{array}{cc}
R(\theta) & 0 \\
0 & R(\theta)
\end{array}\right] \in G \quad \text { and } \quad\left[\begin{array}{cc}
R(\theta) & 0 \\
0 & R(\phi)
\end{array}\right]^{\sigma}=\left[\begin{array}{cc}
R(-\theta) & 0 \\
0 & R(\phi)
\end{array}\right] .
$$

We need to find the normalizers of the finite subgroups of $S^{3}$.

3.1. Lemma. For $\Gamma \subset G$, the normalizer of $\Gamma$ in $G$ is as follows :

$$
\begin{array}{ccccccccc}
\Gamma & : & 1 \text { or } \mathbb{Z}_{2} & \mathbb{Z}_{k}(k>2) & \mathbf{D}_{8}^{*} & \mathbf{D}_{4 k}^{*}(k>2) & \mathbf{T}^{*} & \mathbf{O}^{*} & \mathbf{I}^{*} \\
\text { Normalizer } & : & G & \mathrm{SO}(2) \rtimes_{\mathbb{Z}_{2}} \mathbb{Z}_{4} & \mathbf{O}^{*} & \mathbf{D}_{8 k}^{*} & \mathbf{O}^{*} & \mathbf{O}^{*} & \mathbf{I}^{*}
\end{array}
$$

Proof. Since $\Gamma$ is a closed subgroup, its normalizer $N(\Gamma)$ is also a closed, and hence compact, subgroup of the compact Lie group $S^{3}$. Moreover, $N(\Gamma)$ is a Lie group. If $\operatorname{dim} N(\Gamma)=0$, then $N(\Gamma)$ is finite. Suppose that $\operatorname{dim} N(\Gamma)>0$. The subgroup $N(\Gamma)$ of $S^{3}$ acts on $S^{3}$ freely via left multiplications. Then $N(\Gamma)$ is isomorphic to either $S^{1}, N\left(S^{1}\right)$, or $S^{3}$. See [1, p.153]. Note that $N\left(\mathbb{Z}_{2}\right)=S^{3}$, and if $\Gamma ¥ 1$ or $\mathbb{Z}_{2}$, then $N(\Gamma) \neq S^{3}$.

Consider $\mathbb{Z}_{k}$ with $k>2$. By conjugating if necessary, we may assume that a generator of $\mathbb{Z}_{k}$ is $\mathcal{R}(1 / k)$. Then $\mathcal{R}(\theta) \in G$ commutes with $\mathbb{Z}_{k}$, and conjugation by $\tau$, where

$$
\tau=\left[\begin{array}{cc}
0 & J \\
-J & 0
\end{array}\right] \text { and } J=\left[\begin{array}{cc}
-1 & 0 \\
0 & 1
\end{array}\right],
$$

inverts $\mathbb{Z}_{k}$. Clearly, $\mathrm{SO}(2)=\{\mathcal{R}(\theta): 0 \leq \theta<1\}$ and $\{\tau\}$ generate the normalizer of $\mathbb{Z}_{k}$ for $k>0$. Since $\tau^{2}=-I=\mathcal{R}(1 / 2) \in \mathrm{SO}(2), \tau^{4}=I$. Therefore, $N\left(\mathbb{Z}_{k}\right)=$ $\mathrm{SO}(2) \rtimes_{\mathbb{Z}_{2}} \mathbb{Z}_{4}$. 
For $\Gamma=\mathbf{D}_{4 k}^{*}=\mathbb{Z}_{2 k} \rtimes_{\mathbb{Z}_{2}} \mathbb{Z}_{4}$ with $k>2$, in order for an element of $S^{3}$ to normalize $\mathbf{D}_{4 k}^{*}$, it should normalize the characteristic subgroup $\mathbb{Z}_{2 k}$. Therefore, $N\left(\mathbf{D}_{4 k}^{*}\right)$ is contained in $\mathrm{SO}(2) \rtimes_{\mathbb{Z}_{2}} \mathbb{Z}_{4}$. Now it is easy to see that $N\left(\mathbf{D}_{4 k}^{*}\right)$ is $\mathbb{Z}_{4 k} \rtimes_{\mathbb{Z}_{2}} \mathbb{Z}_{4}=\mathbf{D}_{8 k}^{*}$.

Let $\Gamma=\mathbf{D}_{8}^{*}$. It is well known that $\mathbf{D}_{8}^{*}$ is a normal subgroup of $\mathbf{O}^{*}$. No binary dihedral group $\mathbf{D}_{4 k}^{*}$ can contain $\mathbf{O}^{*}$. Therefore, $N\left(\mathbf{D}_{8}^{*}\right)=\mathbf{O}^{*}$.

The case of $\Gamma=\mathbf{T}^{*}, \mathbf{O}^{*}$, or $\mathbf{I}^{*}$ : If $N(\Gamma)_{0} \cong \mathrm{SO}(2)$, then $N(\Gamma)=\mathrm{SO}(2) \rtimes_{\mathbb{Z}_{2}} \mathbb{Z}_{4}$ $=N(\mathrm{SO}(2))$, since it is the only proper subgroup of $S^{3}$ containing $\mathrm{SO}(2)$. Since $N(\mathrm{SO}(2))$ cannot contain $\Gamma$, this is impossible. Hence $N(\Gamma)$ is a finite group. Since $\mathbf{T}^{*}$ is a normal subgroup of $\mathbf{O}^{*}$, it follows that $N\left(\mathbf{T}^{*}\right)=\mathbf{O}^{*}$. It is easy to see that $N\left(\mathbf{O}^{*}\right)=\mathbf{O}^{*}$ and $N\left(\mathbf{I}^{*}\right)=\mathbf{I}^{*}$.

An element of $G \subset \mathrm{SO}(4)$ is determined by its first column. In fact, there is a natural one-to-one correspondence between $S^{3} \subset \mathbb{R}^{4}$ and $G$, by $\mathbf{v} \leftrightarrow v$, where $v$ is the unique element of $G$ whose first column is $\mathbf{v}$. Furthermore, the action of $a \cdot b^{\sigma} \in \mathrm{SO}(4)$ on $\mathbf{v} \in S^{3}$ corresponds to the matrix product $a \cdot v \cdot b^{-1}$, verifying that the subgroups $G$ and $G^{\sigma}$ correspond to $\ell(G)$ and $r(G) \subset \mathrm{M}(W, G)$. The following diagram is commutative:

$$
\begin{array}{cccccc}
(\ell(G) \times r(G)) \times G & \rightarrow & G & ((a, b), v) & \rightarrow & a \cdot v \cdot b^{-1} \\
\downarrow & & \downarrow & \downarrow & & \downarrow \\
\mathrm{SO}(4) \times S^{3} & \rightarrow & S^{3} & \left(a \cdot b^{\sigma}, \mathbf{v}\right) & \rightarrow & \left(a \cdot b^{\sigma}\right) \mathbf{v}
\end{array}
$$

Note that $\ell(G)$ and $r(G)$ are the same group $G$. We view these as different subgroups of $\operatorname{TOP}(G)$. Also, note that

$$
\begin{gathered}
\ell(G) \times r(G) \\
(a, b) \longrightarrow \mathrm{SO}(4), \\
\quad \longrightarrow \cdot b^{\sigma},
\end{gathered}
$$

is a two-to-one map. In fact, the subgroup $\{ \pm(I, I)\}=\left\{(z, z): z \in \mathbb{Z}_{2}\right\}$, where $\mathbb{Z}_{2}=\ell(G) \cap r(G)$ is exactly the ineffective part of the action of $\ell(G) \times r(G)$ on $G$. All spherical space-form groups can be put into either $\ell(G) \times_{\mathbb{Z}_{2}} r\left(S^{1}\right)$ or $\ell\left(S^{1}\right) \times_{\mathbb{Z}_{2}} r(G)$, where $\mathbb{Z}_{2}=\ell(G) \cap r(G)$ as above. Sometimes a spherical space-form group $\Delta \subset$ $\mathrm{SO}(4)$ lifts to a subgroup of $\ell(G) \times r(G)$. Otherwise, we can take a central extension $\Delta^{*} \subset \ell(G) \times r(G)$ so that $1 \rightarrow \mathbb{Z}_{2} \rightarrow \Delta^{*} \rightarrow \Delta \rightarrow 1$ is exact. We still call such a $\Delta^{*}$ a spherical space-form group.

Let $i=i_{\ell} \times i_{r}: \Delta \hookrightarrow \ell(G) \times r(G)$ be an injective homomorphism. We denote

$$
\begin{array}{ll}
K_{\ell}=\operatorname{Ker}\left(i_{\ell}\right)=\Delta \cap r(G), & K_{r}=\operatorname{Ker}\left(i_{r}\right)=\Delta \cap \ell(G), \\
\Delta_{\ell}=\operatorname{Im}\left(i_{\ell}\right)=\Delta / K_{\ell}, & \Delta_{r}=\operatorname{Im}\left(i_{r}\right)=\Delta / K_{r} .
\end{array}
$$

We shall say that $H$ is a diagonal subgroup of $H_{1} \times H_{2}$ if there exist surjections $f_{1}$ and $f_{2}$ from $H_{1}$ and $H_{2}$ respectively to some abelian group $A$, and $H$ is the kernel of the map $H_{1} \times H_{2} \rightarrow A$ whose restriction to $H_{i}$ is $f_{i}$. More generally, a group $H$ is a diagonal subgroup of $H_{1} \times H_{2}$ if there exist a (not necessarily abelian) group $A$ and surjective homomorphisms $f_{1}: H_{1} \rightarrow A$ and $f_{2}: H_{2} \rightarrow A$ so that $H$ is the pullback of $f_{1}$ and $f_{2}$. That is, $H=\left\{(x, y) \in H_{1} \times H_{2}: f_{1}(x)=f_{2}(y)\right\}$.

The following is known from work of Seifert and Threlfall, see for example, [18, p.224], [15, 4.11], [13, p.111], [12] or [14]. 
3.2. Theorem. Let $\Delta$ be a finite subgroup of $\mathrm{SO}(4)$ acting freely on $S^{3}$. Then one of the following holds. In all cases, $r\left(S^{3}\right)$ and $\ell\left(S^{3}\right)$ can be interchanged.

1. $\Delta=\mathbb{Z}_{n}$ lifts to $\ell\left(S^{3}\right) \times r\left(S^{3}\right)$ as a diagonal subgroup of $\mathbb{Z}_{p} \times \mathbb{Z}_{q}$ so that

$$
\begin{array}{rlrl}
K_{\ell} & =\mathbb{Z}_{p^{\prime}}, & K_{r}=\mathbb{Z}_{q^{\prime}}, \\
\Delta_{\ell}=\mathbb{Z}_{p}, & \Delta_{r}=\mathbb{Z}_{q},
\end{array}
$$

where $p, p^{\prime}, q, q^{\prime}$ are certain integers satisfying $p p^{\prime}=q q^{\prime}=n$.

2. $\Delta=\mathbf{B}^{*} \times \mathbb{Z}_{m}$, where $\mathbf{B}^{*}$ is one of the binary polyhedral groups and $m$ is coprime to the order of $\mathbf{B}^{*}$, lifts to $\ell\left(S^{3}\right) \times r\left(S^{3}\right)$ as

$$
\begin{aligned}
K_{\ell} & =\mathbb{Z}_{m}, & K_{r} & =\mathbf{B}^{*}, \\
\Delta_{\ell} & =\mathbf{B}^{*}, & \Delta_{r} & =\mathbb{Z}_{m} .
\end{aligned}
$$

3. $\Delta$ is an extension of a cyclic group $\mathbb{Z}_{2 n}$ by $\mathbb{Z}_{2}$. Its double covering $\Delta^{*}$ is a diagonal subgroup of $\mathbb{Z}_{4 u} \times \mathbf{D}_{4 v}^{*} \subset \ell\left(S^{3}\right) \times r\left(S^{3}\right)$ of index 2 , where $n=u v$ with $(u, v)=1$ and $u$ even, and $\Delta=\Delta^{*} / \mathbb{Z}_{2}$. Therefore, $\Delta^{*}$ embeds in $\ell\left(S^{3}\right) \times r\left(S^{3}\right)$ as

$$
\begin{array}{rlrl}
K_{\ell} & =\mathbb{Z}_{2 u}, & & K_{r}=\mathbb{Z}_{2 v}, \\
\Delta_{\ell}^{*} & =\mathbf{D}_{4 v}^{*}, & \Delta_{r}^{*}=\mathbb{Z}_{4 u} .
\end{array}
$$

4. $\Delta^{*} \subset \ell\left(S^{3}\right) \times r\left(S^{3}\right)$ is a diagonal subgroup of $\mathbf{T}^{*} \times \mathbb{Z}_{6 m}$ of index 3 , and $\Delta=\Delta^{*} / \mathbb{Z}_{2}$ :

$$
\begin{array}{ll}
K_{\ell}=\mathbb{Z}_{2 m}, & K_{r}=\mathbf{D}_{8}^{*}, \\
\Delta_{\ell}^{*}=\mathbf{D}_{8}^{*} \rtimes \mathbb{Z}_{3}=\mathbf{T}^{*}, & \Delta_{r}^{*}=\mathbb{Z}_{6 m} .
\end{array}
$$

In all cases, $K_{\ell}$ and $K_{r}$ are characteristic subgroups of $\Delta$ or $\Delta^{*}$.

Proof. (1) A cyclic group $\mathbb{Z}_{n}$ in $\mathrm{SO}(4)$ is generated by

$$
\left[\begin{array}{cc}
R\left(\frac{r}{n}\right) & 0 \\
0 & R\left(\frac{1}{n}\right)
\end{array}\right]=\left[\begin{array}{cc}
R\left(\frac{1+r}{2 n}\right) & 0 \\
0 & R\left(\frac{1+r}{2 n}\right)
\end{array}\right] \cdot\left[\begin{array}{cc}
R\left(\frac{1-r}{2 n}\right) & 0 \\
0 & R\left(\frac{1-r}{2 n}\right)
\end{array}\right]^{\sigma}=\mathcal{R}\left(\frac{1+r}{2 n}\right) \cdot \mathcal{R}\left(\frac{1-r}{2 n}\right)^{\sigma}
$$

up to conjugation in $\mathrm{SO}(4)$. Then $\mathbb{Z}_{n}$ acts freely if and only if $r$ satisfies $(r, n)=1$. Furthermore, any two such representations with $r$ and $r^{\prime}$ are conjugate in $O(4)$ if and only if $r \equiv \pm r^{\prime} \bmod n$ or $r r^{\prime} \equiv \pm 1 \bmod n$. Suppose $r$ is even, $0<r<n$. Then $n$ is odd since $(r, n)=1$. Take $r^{\prime}=n-r$. Then $r^{\prime}$ is odd. Therefore, without loss of generality, we may assume that $r$ is odd. Then we define a lift $\mathbb{Z}_{n} \rightarrow \ell(G) \times r(G)$ by

$$
\mathrm{SO}(4) \ni \mathcal{R}\left(\frac{1+r}{2 n}\right) \cdot \mathcal{R}\left(\frac{1-r}{2 n}\right)^{\sigma} \mapsto \quad\left(\mathcal{R}\left(\frac{1+r}{2 n}\right), \mathcal{R}\left(\frac{1-r}{2 n}\right)\right) \in \ell(G) \times r(G) .
$$

Since $r$ is odd, $\left(\mathcal{R}\left(\frac{1+r}{2 n}\right), \mathcal{R}\left(\frac{1-r}{2 n}\right)\right)$ has order exactly $n$ in $\ell\left(S^{3}\right) \times r\left(S^{3}\right)$. Note that if $r \pm 1 \equiv 0 \bmod n$, then $\Delta$ lies completely in one of $\ell(G)$ or $r(G)$. In general, $\Delta$ lies in $\ell(G) \times r(G)$ diagonally.

The order of $\Delta_{\ell}$ and $\Delta_{r}$ are $p=2 n / \operatorname{gcd}(2 n, 1+r)$ and $q=2 n / \operatorname{gcd}(2 n, 1-r)$; and $p^{\prime}=n / p, q^{\prime}=n / q$.

In the following, we indicate the commutative diagram 


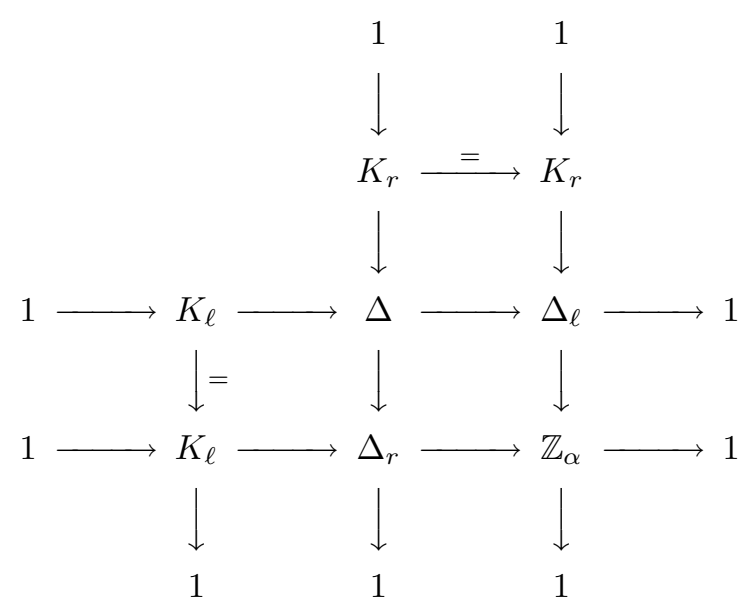

omitting the trivial groups.

Then the group $\mathbb{Z}_{n}$ fits the commutative diagram

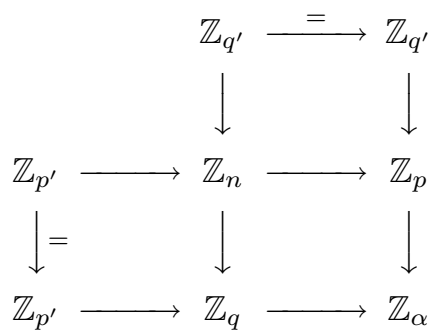

so that $\mathbb{Z}_{n}$ is a diagonal subgroup of $\mathbb{Z}_{p} \times \mathbb{Z}_{q}$ of index $\alpha$, where $\alpha=p q / n$.

(2) The product $\Delta=\mathbf{B}^{*} \times \mathbb{Z}_{m}$ maps into $\ell\left(S^{3}\right) \times r\left(S^{3}\right)$ by $\mathbf{B}^{*} \subset \ell\left(S^{3}\right)$ and $\mathbb{Z}_{m} \subset r\left(S^{3}\right)$ so that it fits the commutative diagram

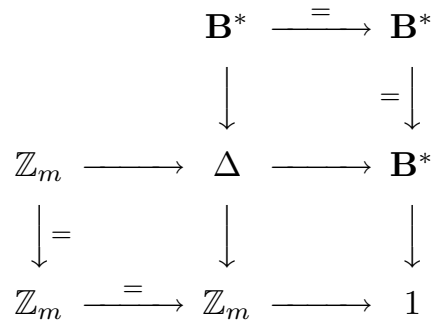

(3) A decomposition $2 n=2 u \cdot v, u=2 w$ with $(2 w, v)=1$, gives rise to an embedding of $\Delta^{*}$ as follows: Let $x, y$ be integers such that $2 w x+v y=1$. Then let $r \equiv 1-4 w x(\equiv-1+2 v y) \bmod 2 n$. Then $r^{2} \equiv 1 \bmod 2 n$. Such an $r$ is called a regular solution of $r^{2} \equiv 1 \bmod 2 n$ of even type associated with the decomposition $2 n=2 u \cdot v, u=2 w . \Delta$ is generated by

$$
C_{2 n}^{(r)}=\left[\begin{array}{cc}
R\left(\frac{r}{2 n}\right) & 0 \\
0 & R\left(\frac{1}{2 n}\right)
\end{array}\right] \quad \text { and } \quad V=\left[\begin{array}{cc}
0 & R\left(\frac{1}{2 u}\right) \\
-I & 0
\end{array}\right]
$$


Then $\Delta^{*}$ fits the diagram

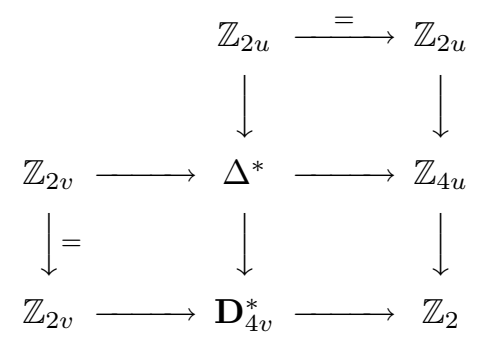

so that $\Delta^{*}$ is a diagonal subgroup of $\mathbb{Z}_{4 u} \times \mathbf{D}_{4 v}^{*} \subset \ell\left(S^{3}\right) \times r\left(S^{3}\right)$ of index 2 .

For example, let $2 n=24$. An even decomposition $24=(2 \cdot 4) \cdot 3,2 w=u=4$, $v=3$, and $(2 w, v)=(4,3)=1$, gives rise to subgroup $\Delta$ of $\mathrm{SO}(4)$ generated by

$$
\left[\begin{array}{cc}
R\left(\frac{17}{24}\right) & 0 \\
0 & R\left(\frac{1}{24}\right)
\end{array}\right] \text { and }\left[\begin{array}{cc}
0 & R\left(\frac{1}{8}\right) \\
-I & 0
\end{array}\right]
$$

Observe that these can be written as

$$
C=\mathcal{R}\left(\frac{3}{8}\right) \cdot \mathcal{R}\left(-\frac{1}{3}\right)^{\sigma} \quad \text { and } \quad V=\mathcal{R}\left(-\frac{1}{16}\right) \cdot W^{\sigma},
$$

where $W$ is a matrix such that $W^{2}=-I, W \mathcal{R}\left(-\frac{1}{3}\right) W^{-1}=\mathcal{R}\left(\frac{1}{3}\right)$, and it commutes with every other matrix. Therefore, $K_{r}=\mathbb{Z}_{8}$ and $K_{\ell}=\mathbb{Z}_{6}$ are generated by $C^{3}$ and $C^{5} V^{-2}$, respectively.

Remark. An odd decomposition of $2 n$ gives rise to a product embedding which is case (2). For more details. the reader is referred to the paper [14], but one should beware of many typographical errors in that paper. Specifically, in page 149 line $-17, u=2 v$ should read $u=2 w$. Also in the same page, line -10 , all $n$ 's should read $u$ 's. ${ }^{1}$

(4) The group $\Delta^{*}$ fits the commutative diagram

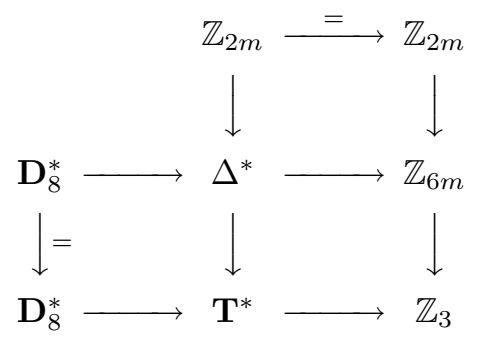

so that it is a diagonal subgroup of $\mathbf{T}^{*} \times \mathbb{Z}_{6 m}$ of index 3 . Of course, $\Delta=\Delta^{*} / \mathbb{Z}_{2}$.

Finally, from the list, it is clear that all $K_{\ell}$ and $K_{r}$ are characteristic in $\Delta$ or $\Delta^{*}$.

Let $Q^{\prime}$ be an extension of a non-cyclic binary polyhedral group $\mathbf{B}^{*}$ by $Q$. Consider the following condition on $Q^{\prime}$.

\footnotetext{
${ }^{1}$ If you happen to be using the Russian original of [14], the same errors appear on page 135, lines 2 and 9 .
} 


\section{Condition (B).}

1. If $\mathbf{B}^{*}=\mathbf{D}_{8}^{*}, Q^{\prime}$ is either a product $\mathbf{D}_{8}^{*} \times Q$, a diagonal subgroup of $\mathbf{D}_{16}^{*} \times Q$ of index 2 , a diagonal subgroup of $\mathbf{T}^{*} \times Q$ of index 3 , or a diagonal subgroup of $\mathbf{O}^{*} \times Q$ of index 6 .

2. If $\mathbf{B}^{*}=\mathbf{D}_{4 k}^{*}(k>2)$ or $\mathbf{T}^{*}$, then $Q^{\prime}$ is either $\mathbf{B}^{*} \times Q$ or a diagonal subgroup of $\mathbf{D}_{8 k}^{*} \times Q$ or $\mathbf{O}^{*} \times Q$ of index 2 , respectively.

3. If $\mathbf{B}^{*}=\mathbf{O}^{*}$ or $\mathbf{I}^{*}$, then $Q^{\prime}=\mathbf{B}^{*} \times Q$.

From Main Lemma 2.2, we have

3.3. Lemma (Non-cyclic). Let $i: \Delta \hookrightarrow G$ be a finite non-cyclic binary polyhedral group, $\rho: Q \rightarrow \operatorname{TOP}(W)$ be an action on a connected space $W$; and let $1 \rightarrow \Delta \rightarrow$ $\Pi \rightarrow Q \rightarrow 1$ be exact. The following are equivalent:

(1) There exists a homomorphism $\bar{\theta}: \Pi \rightarrow \mathrm{M}(W, G) \rtimes \operatorname{TOP}(W)$ extending $i$ : $\Delta \hookrightarrow r(G) \subset \mathrm{M}(W, G)$ and compatible with $\rho$.

(2) There exists a homomorphism $\bar{\theta}: \Pi \rightarrow G \times \operatorname{TOP}(W)$ compatible with $i$ and $\rho$.

(3) There exists a homomorphism $\widetilde{i}: \Pi \rightarrow G$ extending $i$.

(4) $I$ satisfies the condition $(\mathbf{B})$.

In each of the cases, there exists only one homomorphism $\widetilde{i}$ defined by projecting $\Pi$ onto the first factor of the product and then followed by the inclusion of the first factor into $G$. The homomorphism $\bar{\theta}$ is injective if and only if $\rho$ is injective.

Proof. Suppose there exists $\bar{\theta}: \Pi \rightarrow \mathrm{M}(W, G) \rtimes \mathrm{TOP}(W)$. For any $(\eta, h) \in$ $\mathrm{M}(W, G) \rtimes \operatorname{TOP}(W)$,

$$
(\eta, h)(z, 1)(\eta, h)^{-1}=\left(\eta \cdot z \cdot \eta^{-1}, 1\right)
$$

for $z \in r(G)$. This implies that if $(\eta, h) \in \bar{\theta}(\Pi)$, then $\eta: W \rightarrow G$ must have values in $N_{G}(\Delta)$. In other words, $\bar{\theta}: \Pi \rightarrow \mathrm{M}\left(W, N_{G}(\Delta)\right) \rtimes \operatorname{TOP}(W)$. Since $\Delta$ is not cyclic, $N_{G}(\Delta)$ is discrete. Moreover, since $W$ is connected, every continuous map into $N_{G}(\Delta)$ is constant so that $\mathrm{M}\left(W, N_{G}(\Delta)\right)=N_{G}(\Delta)$. Note also that the action of $\operatorname{TOP}(W)$ on $N_{G}(\Delta) \subset r(G)$ is trivial. Consequently, any homomorphism $\bar{\theta}: \Pi \rightarrow \mathrm{M}(W, G) \rtimes \mathrm{TOP}(W)$ has image in $r(G) \times \operatorname{TOP}(W) \subset \mathrm{M}(W, G) \rtimes \mathrm{TOP}(W)$, and such a homomorphism exists if and only if there exists a homomorphism $\widetilde{i}$ : $\Pi \rightarrow N_{G}(\Delta) \subset G$ extending $i$.

Now the image $\widetilde{i}(\Pi)$ is isomorphic to either $\Delta$ or a normal subgroup $S$ of $N_{G}(\Delta)$ containing $\Delta$. If $\widetilde{i}(\Pi) \cong \Delta$, then $\Pi$ is a product $\Delta \times Q$. Otherwise, $\Pi$ is a diagonal subgroup of $S \times Q$. This happens only when $\Delta=\mathbf{D}_{4 k}^{*}$ or $\mathbf{T}^{*}$, see 3.1 .

To treat the case when $\Delta$ is cyclic, we use cohomology arguments. We shall use the first and second group cohomology, but they have non-abelian coefficients. The first cohomology arises from crossed homomorphisms. With the same notation as in Lemma 3.3, any homomorphism $\bar{\theta}: \Pi \rightarrow \mathrm{M}(W, G) \rtimes \mathrm{TOP}(W)$ compatible with $\rho: Q \rightarrow \operatorname{TOP}(W)$ yields a crossed homomorphism $\lambda: \Pi \rightarrow \mathrm{M}(W, G)$. That is,

$$
\lambda(\alpha \beta)=\lambda(\alpha) \cdot \bar{\alpha} \lambda(\beta)=\lambda(\alpha) \cdot \lambda(\beta) \circ \rho(\bar{\alpha})^{-1},
$$

where $\bar{\alpha} \in Q$ is the image of $\alpha \in \Pi$. Conversely, any such a crossed homomorphism $\lambda$ gives rise to a homomorphism $\bar{\theta}$. If $\mathrm{M}(W, G)$ is replaced by $r(G)$, the action of $\operatorname{TOP}(W)$ on $r(G)$ is trivial, so that the crossed homomorphism becomes a homomorphism. 
For a group $Q$, a commutative group $A$ and a homomorphism $\phi: Q \rightarrow \operatorname{Aut}(A)$, each congruence class of extensions of the form $0 \rightarrow A \rightarrow \Pi \rightarrow Q \rightarrow 1$ with abstract kernel $\phi$ is represented by an element of $H_{\phi}^{2}(Q ; A)$. We denote the element of $H_{\phi}^{2}(Q ; A)$ which is represented by this extension by [I]. See [11]. If $\phi$ is obvious from the context, we suppress it and write $H_{\phi}^{2}(Q ; A)$ as $H^{2}(Q ; A)$.

3.4. Lemma (Cyclic). Let $i: \Delta=\mathbb{Z}_{n} \hookrightarrow r(G), n>2$ (so that $N_{G}(\Delta)=$ $\left.\mathrm{SO}(2) \rtimes_{\mathbb{Z}_{2}} \mathbb{Z}_{4}\right)$, let $\rho: Q \hookrightarrow \operatorname{TOP}(W)$ be an effective properly discontinuous action on a simply connected space $W$; and let $1 \rightarrow \Delta \rightarrow \Pi \rightarrow Q \rightarrow 1$ be exact. The following are equivalent:

1. There exists an injective homomorphism $\bar{\theta}: \Pi \rightarrow \overline{\mathcal{U}}=\mathrm{M}(W, G) \rtimes \operatorname{TOP}(W)$ extending $i: \Delta \hookrightarrow r(G) \subset \mathrm{M}(W, G)$ and compatible with $\rho$.

2. There exists a crossed homomorphism $\tilde{i}: \Pi \rightarrow \mathrm{M}\left(W, \mathrm{SO}(2) \rtimes_{\mathbb{Z}_{2}} \mathbb{Z}_{4}\right)$ extending $i$.

If 1 holds, then either the subgroup $\Delta$ is central or elements of $\Pi$ must act as inversion on $\Delta$. If $\Delta$ is central, the above is also equivalent to

3. There exists an exact sequence $1 \rightarrow \mathbb{Z} \rightarrow \widetilde{\Pi} \rightarrow Q \rightarrow 1$ so that $1 \rightarrow \Delta \rightarrow \Pi \rightarrow$ $Q \rightarrow 1$ is its natural image.

Suppose $\Delta$ is central, and 3 holds. Then $\bar{\theta}$ has image in $\operatorname{SO}(2) \times \operatorname{TOP}(W)$ (and consequently, $i$ extends to a homomorphism $\Pi \rightarrow \mathrm{SO}(2))$ if and only if

4. The image of $[\widetilde{\Pi}]$ under the homomorphism $j_{*}: H^{2}(Q ; \mathbb{Z}) \rightarrow H^{2}(Q ; \mathbb{R})$ induced by the inclusion map $j: \mathbb{Z} \hookrightarrow \mathbb{R}$ lies in $n \cdot \operatorname{Image}\left(j_{*}\right) \subset H^{2}(Q ; \mathbb{R})$.

Proof. A homomorphism $\bar{\theta}: \Pi \rightarrow \mathrm{M}(W, G) \rtimes \mathrm{TOP}(W)$ induces a crossed homomorphism $\Pi \rightarrow \mathrm{M}(W, G)$ which necessarily has values in $\mathrm{M}\left(W, \mathrm{SO}(2) \rtimes_{\mathbb{Z}_{2}} \mathbb{Z}_{4}\right.$ ) (recall that $\left.N_{G}(\Delta)=\mathrm{SO}(2) \rtimes_{\mathbb{Z}_{2}} \mathbb{Z}_{4}\right)$, and conversely, such a crossed homomorphism gives rise to a homomorphism $\bar{\theta}: \Pi \rightarrow \mathrm{M}(W, G) \rtimes \mathrm{TOP}(W)$. Thus 1 and 2 are equivalent.

We show that $1 \Longleftrightarrow 3$. Suppose (1) holds. Then $\bar{\theta}$ must have image in $\mathrm{M}\left(W, \mathrm{SO}(2) \rtimes_{\mathbb{Z}_{2}} \mathbb{Z}_{4}\right) \rtimes \mathrm{TOP}(W)$. (See the proof of Lemma 3.3.) This implies the abstract kernel $Q \rightarrow \operatorname{Aut}(\Delta)$ is either trivial or has image $\mathbb{Z}_{2}$ whose non-trivial element is the inversion on $\Delta$.

Suppose $\Delta$ is central. View $\mathrm{SO}(2)$ as $\mathbb{R} / n \mathbb{Z}$, and take the pullback $\widetilde{\Pi}$ of the two homomorphisms $\bar{\theta}: \Pi \rightarrow \mathrm{M}(W, \mathrm{SO}(2)) \rtimes \operatorname{TOP}(W)$ and $\mathrm{M}(W, \mathbb{R}) \rtimes \operatorname{TOP}(W) \rightarrow$ $\mathrm{M}(W, \mathrm{SO}(2)) \rtimes \mathrm{TOP}(W)$, to get

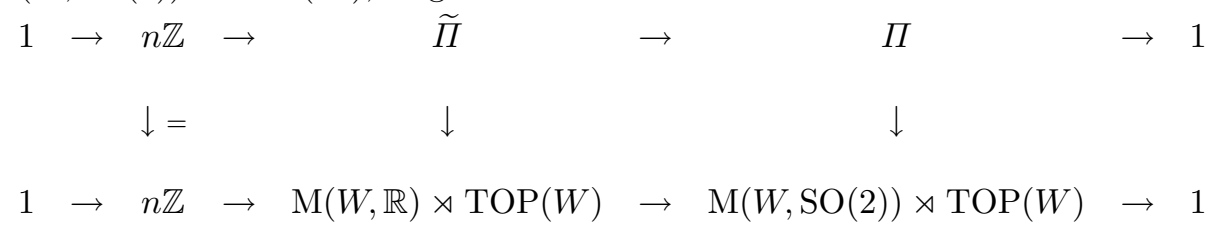

Simple connectivity of $W$ assures that the bottom sequence is exact. By restricting the above diagram to the subgroup $\mathbb{Z}_{n}=\Delta \subset \Pi$, we get a subgroup $A \subset \widetilde{\Pi}$ and a commutative diagram

$$
\begin{aligned}
& 1 \rightarrow n \mathbb{Z} \rightarrow A \rightarrow \mathbb{Z}_{n} \rightarrow 1 \\
& 1 \rightarrow n \mathbb{Z} \rightarrow \mathbb{R} \rightarrow \mathrm{SO}(2) \rightarrow 1
\end{aligned}
$$


This implies $A$ is torsion free, and hence $A \cong \mathbb{Z}$. Since $\widetilde{\Pi} / A \cong Q$, we have a desired extension $1 \rightarrow \mathbb{Z}=A \rightarrow \widetilde{\Pi} \rightarrow Q \rightarrow 1$.

Conversely, suppose there exists an exact sequence $1 \rightarrow \mathbb{Z} \rightarrow \widetilde{\Pi} \rightarrow Q \rightarrow 1$ which maps down to $1 \rightarrow \Delta \rightarrow \Pi \rightarrow Q \rightarrow 1$ naturally. We shall first map $\widetilde{\Pi}$ into $\mathrm{M}(W, \mathbb{R}) \rtimes \operatorname{TOP}(W)$, and then it will induce a desired homomorphism $\Pi \rightarrow \mathrm{M}(W, \mathrm{SO}(2) \rtimes \mathrm{TOP}(W)) \subset \mathrm{M}(W, G) \rtimes \mathrm{TOP}(W)$ via $\mathrm{SO}(2) \subset G$. In order to find a homomorphism $\bar{\theta}^{\prime}: \widetilde{\Pi} \rightarrow \mathrm{M}(W, \mathbb{R}) \rtimes \mathrm{TOP}(W)$, we employ a cohomological argument. The existence of a homomorphism $\bar{\theta}^{\prime}$ which makes the diagram

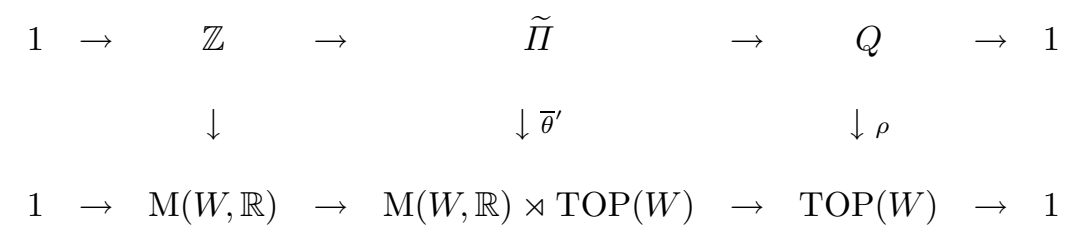

commutative is equivalent to saying that $[\widetilde{\Pi}] \in H^{2}(Q ; \mathbb{Z})$ maps to $[\mathrm{M}(W, \mathbb{R}) \rtimes$ $Q]=0 \in H^{2}(Q ; \mathrm{M}(W, \mathbb{R}))$ under the homomorphism $H^{2}(Q ; \mathbb{Z}) \rightarrow H^{2}(Q ; \mathrm{M}(W, \mathbb{R}))$ induced by the coefficient homomorphism $\mathbb{R} \hookrightarrow \mathrm{M}(W, \mathbb{R})$. However, it is known [2] that the group $H^{2}(Q ; \mathrm{M}(W, \mathbb{R}))$ is 0 when the action $\rho: Q \rightarrow \operatorname{TOP}(W)$ is properly discontinuous. Now divide out $\widetilde{\Pi} \rightarrow \mathrm{M}(\mathrm{W}, \mathbb{R}) \rtimes \mathrm{TOP}(W)$ by $n \mathbb{Z}$ to get a homomorphism of $\Pi \rightarrow \mathrm{M}(W, \mathrm{SO}(2)) \rtimes \mathrm{TOP}(W)$. Since $\mathrm{SO}(2) \subset G$, we have obtained a desired $\bar{\theta}: \Pi \rightarrow \mathrm{M}(W, G) \rtimes \mathrm{TOP}(W)$. This establishes the equivalence of 1,2 and 3 when $\Delta$ is central.

To prove the final claim, suppose $\Delta$ is central, and 3 holds. The commutative diagram (3.4.1) of trivial $Q$-modules yields a commutative diagram

$$
\begin{array}{ccccccc}
H^{2}(Q ; n \mathbb{Z}) & \stackrel{j}{\rightarrow} & H^{2}(Q ; \mathbb{Z}) & \stackrel{p}{\rightarrow} & H^{2}(Q ; \Delta) & \rightarrow & H^{3}(Q ; n \mathbb{Z}) \\
\| & & \downarrow j * & & \downarrow \bar{j}_{*} & & \| \\
H^{2}(Q ; n \mathbb{Z}) & \stackrel{j^{\prime}}{\longrightarrow} & H^{2}(Q ; \mathbb{R}) & \stackrel{p^{\prime}}{\longrightarrow} & H^{2}(Q ; \operatorname{SO}(2)) & \rightarrow & H^{3}(Q ; n \mathbb{Z})
\end{array}
$$

Suppose $\bar{\theta}$ has image in $G \times \operatorname{TOP}(W)$. Then, in fact, $\Pi$ maps into $\mathrm{SO}(2) \times Q$. (We identify $Q$ with $\rho(Q)$.) This implies $\bar{j}^{*}[\Pi]=0=[\mathrm{SO}(2) \times Q]$. Since $p[\widetilde{\Pi}]=[\Pi]$, we have $p^{\prime} j_{*}[\widetilde{\Pi}]=\bar{j}^{*} p[\widetilde{\Pi}]=\bar{j}^{*}[\Pi]=0$ by the commutativity of the second square of the diagram. By exactness, there exists $a \in H^{2}(Q ; n \mathbb{Z})$ such that $j^{\prime}(a)=j_{*}[\widetilde{\Pi}]$. However, $j^{\prime}$ is just $\otimes \mathbb{R}$ and is the same as the composite $H^{2}(Q ; \mathbb{Z}) \stackrel{n}{\longrightarrow} H^{2}(Q ; \mathbb{Z}) \stackrel{\otimes \mathbb{R}}{\longrightarrow}$ $H^{2}(Q ; \mathbb{R})$. Consequently, $j_{*}[\widetilde{\Pi}]$ lies in $n \cdot \operatorname{Image}\left(j_{*}\right) \subset H^{2}(Q ; \mathbb{R})$.

Conversely, suppose $j_{*}[\tilde{\Pi}]$ lies in $n \cdot \operatorname{Image}\left(j_{*}\right) \subset H^{2}(Q ; \mathbb{R})$. Then it is in the image of $j^{\prime}$ by the above argument. By exactness, $p^{\prime} j_{*}[\widetilde{\Pi}]=0 \in H^{2}(Q ; \mathrm{SO}(2))$. The commutativity of the second square again implies $\bar{j}^{*}[\Pi]=0$, which is equivalent to saying that $\Pi$ maps into $\mathrm{SO}(2) \times Q$. Since $\mathrm{SO}(2) \subset G$, we have obtained a desired $\Pi \rightarrow G \times Q \subset G \times \operatorname{TOP}(W)$.

Note that the above statement 3 is not true if $\Delta$ is not central. For example, suppose $\Pi=\mathbf{D}_{4 n}^{*}=\mathbb{Z}_{2 n} \rtimes_{\mathbb{Z}_{2}} \mathbb{Z}_{4}$, so that $1 \rightarrow \mathbb{Z}_{2 n} \rightarrow \mathbf{D}_{4 n}^{*} \rightarrow \mathbb{Z}_{2} \rightarrow 1$ is exact. There is no exact sequence $1 \rightarrow \mathbb{Z} \rightarrow \widetilde{\Pi} \rightarrow \mathbb{Z}_{2} \rightarrow 1$ which maps to the above exact sequence. Furthermore, even if there were such an exact sequence, the statement 4 
will not work. In fact, $\mathrm{SO}(2) \rtimes_{\mathbb{Z}_{2}} \mathbb{Z}_{4}$ represents a non-zero element of $H^{2}\left(\mathbb{Z}_{2} ; \mathrm{SO}(2)\right)$, and no element of $H^{2}\left(\mathbb{Z}_{2} ; \mathbb{R}\right)=0$ maps to $\left[\mathrm{SO}(2) \rtimes_{\mathbb{Z}_{2}} \mathbb{Z}_{4}\right] \in H^{2}\left(\mathbb{Z}_{2} ; \mathrm{SO}(2)\right)$.

Let $\Delta$ be a spherical space-form group, $\rho: Q \rightarrow \mathrm{TOP}(W)$ a properly discontinuous action; and let $1 \rightarrow \Delta \rightarrow \Pi \rightarrow Q \rightarrow 1$ be exact. Suppose there exists a homomorphism $\Pi \rightarrow \widetilde{\mathcal{U}}$ with $i=i_{\ell} \times i_{r}: \Delta \hookrightarrow \ell(G) \times r(G)$. When $i_{\ell}$ and $i_{r}$ are interchanged, there may not exist such a homomorphism $\Pi \rightarrow \widetilde{\mathcal{U}}$. Recall that at least one of $\Delta_{\ell}$ or $\Delta_{r}$ is cyclic. The following lemma shows that putting a cyclic one in $\Delta_{r}$ rather than in $\Delta_{\ell}$ gives more flexibility for constructing a homomorphism of $\Pi$ into $\widetilde{\mathcal{U}}$. See Examples 4.8 and 4.9 .

3.5. Lemma. Let $i=i_{\ell} \times i_{r}: \Delta \hookrightarrow \ell(G) \times r(G)$ be a spherical space-form group. Let $\rho: Q \rightarrow \operatorname{TOP}(W)$ be a properly discontinuous action; and let $1 \rightarrow \Delta \rightarrow$ $\Pi \rightarrow Q \rightarrow 1$ be exact. Suppose $\Pi$ embeds into $\widetilde{\mathcal{U}}$ so that the diagram (Seifert) below in 3.6 commutes with $\Delta_{\ell}$ cyclic and $\Delta_{r}$ non-cyclic. Then $\theta$ has image in $(\ell(G) \times r(G)) \times \operatorname{TOP}(W)$. Therefore, $\Pi$ can be embedded into $\widetilde{\mathcal{U}}$ also with $i_{\ell}$ and $i_{r}$ interchanged.

Proof. The homomorphism $\theta: \Pi \rightarrow \widetilde{\mathcal{U}}$ induces a homomorphism $\bar{\theta}: \Pi \rightarrow \mathrm{M}(W, G)$ $\rtimes \operatorname{TOP}(W)$. However, since $i_{r}(\Delta)$ is a non-cyclic polyhedral group, $\bar{\theta}$ must have image in $r(G) \times \operatorname{TOP}(W)$ by $3.3(2)$. Consequently, $\theta$ has image in $(\ell(G) \times r(G)) \times$ $\operatorname{TOP}(W)$.

Let $1 \rightarrow \mathbb{Z}_{n} \rightarrow A^{\prime} \rightarrow Q \rightarrow 1$ be exact; and $\rho: Q \rightarrow \mathrm{TOP}(W)$ be an action.

Conditions (C1) and (C2) :

(C1) When $i_{r}: \mathbb{Z}_{n} \hookrightarrow \mathrm{SO}(2) \hookrightarrow r\left(S^{3}\right)$ : There exists a homomorphism $\bar{\theta}: Q^{\prime} \rightarrow$ $\mathrm{M}\left(W, S^{3}\right) \circ \operatorname{TOP}(W)$ extending $i_{r}$. If $n>2$, then this reduces to: there exists a crossed homomorphism $\bar{\theta}: Q^{\prime} \rightarrow \mathrm{M}\left(W, \mathrm{SO}(2) \circ_{\mathbb{Z}_{2}} \mathbb{Z}_{4}\right)$ extending $i$. If $\Delta$ is central and $n>2$, this is equivalent to the existence of an exact sequence $1 \rightarrow \mathbb{Z} \rightarrow Q^{\prime \prime} \rightarrow Q \rightarrow 1$ whose $\bmod n$ reduction is $1 \rightarrow \Delta \rightarrow Q^{\prime} \rightarrow Q \rightarrow 1$.

(C2) When $i_{\ell}: \mathbb{Z}_{n} \hookrightarrow \mathrm{SO}(2) \hookrightarrow \ell\left(S^{3}\right)$ : There exists a homomorphism $\tilde{i}: Q^{\prime} \rightarrow S^{3}$ extending $i_{\ell}$. If $n>2$, then this is equivalent to: there exists a homomorphism $Q^{\prime} \rightarrow \mathrm{SO}(2) \circ_{\mathbb{Z}_{2}} \mathbb{Z}_{4}$ extending $\mathbb{Z}_{n} \hookrightarrow \mathrm{SO}(2)$.

We combine the ideas from the previous two lemmas, and state the final result. This will be used in the next section. We use the conditions (B) in 3.3 for binary polyhedral groups, (C1) and (C2) for cyclic groups.

3.6. Theorem. Let $i=i_{\ell} \times i_{r}: \Delta \hookrightarrow \ell(G) \times r(G)$ be a spherical space-form group; $\rho: Q \hookrightarrow \operatorname{TOP}(W)$ an effective properly discontinuous action; and let $1 \rightarrow \Delta \rightarrow \Pi \rightarrow Q \rightarrow 1$ be exact. Necessary and sufficient conditions that there exists an injective homomorphism $\theta: \Pi \rightarrow \widetilde{\mathcal{U}}$ which makes the diagram

(Seifert)

$$
\begin{aligned}
& 1 \rightarrow \Delta \quad \rightarrow \quad \Pi \rightarrow \quad Q \quad Q \quad \rightarrow 1 \\
& \downarrow i \quad \downarrow \theta \quad \downarrow \rho \\
& 1 \rightarrow \ell(G) \times \mathrm{M}(W, G) \rightarrow \widetilde{\mathcal{U}} \rightarrow \operatorname{TOP}(W) \rightarrow 1
\end{aligned}
$$

commutative are :

1. If $\Delta_{\ell}$ is cyclic and $\Delta_{r}$ is not cyclic: $\Pi / K_{\ell}$ satisfies the condition $(\mathbf{C 2})$, and $\Pi / K_{r}$ satisfies the condition $(\mathbf{B})$. 
2. If $\Delta_{r}$ is cyclic and $\Delta_{\ell}$ is not cyclic: $\Pi / K_{\ell}$ satisfies the condition $(\mathbf{B})$, and $\Pi / K_{r}$ satisfies the condition $(\mathbf{C 1})$.

3. If $\Delta_{r}$ and $\Delta_{\ell}$ are both cyclic: $\Pi / K_{\ell}$ satisfies the condition $(\mathbf{C 2})$, and $\Pi / K_{r}$ satisfies the condition $(\mathbf{C 1})$.

In 2 or 3 , if, furthermore, $\Pi / K_{r}$ satisfies the condition (C2), then $\Pi$ embeds into $\operatorname{Isom}(G) \times \operatorname{TOP}(W)$.

Proof. If $\theta$ exists, it induces homomorphisms $\widetilde{i}: \Pi \rightarrow \ell(G)$ extending $i_{\ell}: \Delta \rightarrow \ell(G)$, and $\bar{\theta}: \Pi \rightarrow \mathrm{M}(W, G) \rtimes \operatorname{TOP}(W)$ extending $i_{r}: \Delta \rightarrow r(G) \subset \mathrm{M}(W, G)$ and compatible with $\rho$.

Conversely, suppose $\Pi$ satisfies the conditions stated in the theorem. Recall that $K_{\ell}$ and $K_{r}$ are characteristic in $\Delta$, and are normal in $\Pi$. We apply Lemmas 3.3 and 3.4 , for the exact sequences $1 \rightarrow \Delta_{\ell} \rightarrow \Pi / K_{\ell} \rightarrow Q \rightarrow 1$ and $1 \rightarrow \Delta_{r} \rightarrow \Pi / K_{r} \rightarrow$ $Q \rightarrow 1$ to get homomorphisms $\tilde{i}: \Pi \rightarrow \ell(G)$ extending $i_{\ell}: \Delta \rightarrow \ell(G)$, and $\bar{\theta}: \Pi \rightarrow$ $\mathrm{M}(W, G) \rtimes \mathrm{TOP}(W)$ extending $i_{r}: \Delta \hookrightarrow r(G) \subset \mathrm{M}(W, G)$ and compatible with $\rho$. Then, applying the Main Lemma 2.2 (combining these two homomorphisms), we get a desired homomorphism $\Pi \rightarrow \ell(G) \times[\mathrm{M}(W, G) \rtimes \mathrm{TOP}(W)]=\widetilde{\mathcal{U}}$.

3.7. Example. Let $\Delta=\mathbb{Z}_{n k}, \Pi=\mathbf{D}_{2 n k}$, so that $Q=\mathbb{Z}_{2}$. Suppose $\Delta \subset \ell(G) \times$ $r(G)$ and $Q$ acts on $S^{2}$ by $180^{\circ}$ rotation around the poles. This $\Pi$ cannot be embedded into $\tilde{\mathcal{U}}$ nor $\mathcal{U}$. If $K_{\ell}=\mathbb{Z}_{n}$, the exact sequence $1 \rightarrow \mathbb{Z}_{k} \rightarrow \mathbf{D}_{2 k} \rightarrow \mathbb{Z}_{2} \rightarrow 1$ cannot map into $\ell(G)$, unless $k=1$. Suppose $k=1$, so that $K_{r}=\mathbb{Z}_{k}=1$. Then the exact sequence $1 \rightarrow \mathbb{Z}_{n} \rightarrow \mathbf{D}_{2 n} \rightarrow \mathbb{Z}_{2} \rightarrow 1$ must map into $\mathrm{M}(W, G)$. However, at the south pole $S, \mathbf{D}_{2 n}$ must map into $\mathrm{M}(\{S\}, G) \cong G$, which is not possible. Consequently, $\mathbf{D}_{2 n k}$ cannot map into $\mathcal{U}$ nor $\widetilde{\mathcal{U}}$.

\section{When $W$ IS 2-DIMENSIONAL}

In this section, we specialize to the case where $W$ is 2-dimensional. In fact, we shall restrict to $W=\mathbb{R}^{2}$ or $W=\mathbf{H}^{2}$, and the action of $Q$ on $W$ will be via orientation-preserving isometries, so that $\rho: Q \rightarrow \operatorname{Isom}_{0}(W)$. We may present a cocompact 2-dimensional orientation-preserving Riemannian orbifold group as follows:

$$
Q=\left\langle\bar{x}_{1}, \cdots, \bar{x}_{g}, \bar{y}_{1}, \cdots, \bar{y}_{g}, \bar{w}_{1}, \cdots, \bar{w}_{p} \mid \bar{w}_{j}^{\alpha_{j}}=1, \prod_{j=1}^{p} \bar{w}_{j} \prod_{i=1}^{g}\left[\bar{x}_{i}, \bar{y}_{i}\right]=1\right\rangle
$$

for $p \geq 0, g \geq 0$ and all $\alpha_{j} \geq 2$. If $g=0$, we require, in addition, that $p \geq 3$. The Euler characteristic of $Q$ is defined as

$$
\chi(Q)=(2-2 g)-\sum_{j=1}^{p}\left(1-\frac{1}{\alpha_{j}}\right) .
$$

Then $Q$ acts properly on $W$ with compact quotient, where $W$ is $S^{2}, \mathbb{R}^{2}$, or $\mathbf{H}^{2}$ according as $\chi(Q)>0,=0$, or $<0$, respectively. In fact, any group acting properly discontinuously and effectively on $S^{2}, \mathbb{R}^{2}$, or $\mathbf{H}^{2}$ preserving orientation and with compact quotient is isomorphic to such a $Q$ and has branching indices equal to the $\alpha_{j}$ 's. We shall restrict ourselves to $\chi(Q) \leq 0$, to insure that $W=\mathbb{R}^{2}$ or $\mathbf{H}^{2}$. Then $Q$ is infinite and has trivial center unless $Q=\mathbb{Z}^{2}$. The latter case occurs only if $g=1$ and $p=0$. 
The embedding problem for $\Delta$ almost always has a cyclic component. The cyclic groups need the most analysis. Consequently, much of present section is devoted to studying when there exists a homomorphism $\widehat{Q} \rightarrow \mathrm{SO}(2)$ which is injective on $\mathbb{Z}_{n}$, for a central extension

$$
1 \rightarrow \mathbb{Z}_{n} \rightarrow \widehat{Q} \rightarrow Q \rightarrow 1
$$

with $\chi(Q) \leq 0$.

Associated with each $Q$ are groups with presentations of the form

$(\widetilde{\mathbf{Q}}) \quad \widetilde{Q}=\left\langle\tilde{x}_{1}, \cdots, \tilde{x}_{g}, \tilde{y}_{1}, \cdots, \tilde{y}_{g}, \tilde{w}_{1}, \cdots, \tilde{w}_{p}, \tilde{z}\right| \tilde{z}$ central,,$\tilde{w}_{j}^{\alpha_{j}}=\tilde{z}^{-\beta_{j}}$,

$$
\left.\prod_{j=1}^{p} \tilde{w}_{j} \prod_{i=1}^{g}\left[\tilde{x}_{i}, \tilde{y}_{i}\right]=\tilde{z}^{b}\right\rangle
$$

$(\widehat{\mathbf{Q}}) \widehat{Q}=\left\langle x_{1}, \cdots, x_{g}, y_{1}, \cdots, y_{g}, w_{1}, \cdots, w_{p}, z\right| z$ central, $\quad z^{n}=1, w_{j}^{\alpha_{j}}=z^{-\beta_{j}}$,

$$
\left.\prod_{j=1}^{p} w_{j} \prod_{i=1}^{g}\left[x_{i}, y_{i}\right]=z^{b}\right\rangle .
$$

4.1. Lemma. Suppose $\chi(Q) \leq 0$. Then

1. A group $Q^{\prime}$ has a presentation of the form $(\widetilde{\mathbf{Q}})$ if and only if it is a central extension of $\mathbb{Z}=\langle\tilde{z}\rangle$ by $Q$.

2. A group $Q^{\prime \prime}$ has a presentation of the form $(\widehat{\mathbf{Q}})$ with $0 \leq \beta_{j}<n$ and $0 \leq b<n$ if and only if it is a central extension of $\mathbb{Z}_{n}=\langle z\rangle$ by $Q$.

3. The $\bmod n$ reduction homomorphism $H^{2}(Q ; \mathbb{Z}) \rightarrow H^{2}\left(Q ; \mathbb{Z}_{n}\right)$ maps $\left[Q^{\prime}\right]$ to $\left[Q^{\prime \prime}\right]$.

Proof. It is well known that the group $Q^{\prime}$ is the fundamental group of a Seifert orbifold group with infinite center, and the element $\tilde{z} \in Q^{\prime}$ generates an infinite cyclic group so that $Q^{\prime}$ is a central extension of $\mathbb{Z}$ by $Q$. See [7] or [6]. Then it is clear that $Q^{\prime \prime}$ is a central extension of the cyclic group generated by $z$, by the group $Q$. If $Q$ is centerless, then the center of $Q^{\prime \prime}$ is the group generated by $z$. If $Q=\mathbb{Z}^{2}$, then the presentation is just $\langle x, y, z|[x, y]=z^{b}, z^{n}=1, z$ central $\rangle$. We can, without loss of generality, assume that $0 \leq \beta_{j}<n$ and $b<n$.

One can define a natural homomorphism $Q^{\prime} \rightarrow Q^{\prime \prime}$ by sending the generators $\tilde{x}_{i} \mapsto x_{i}$, etc. The kernel of this homomorphism is $n \mathbb{Z}$, since all we do to obtain $Q^{\prime \prime}$ is to add the relation $z^{n}=1$. Therefore, $Q^{\prime \prime}=Q^{\prime} / n \mathbb{Z}$, and the subgroups $\mathbb{Z}_{n}$ and $\mathbb{Z}$ generated by $z$ and $\tilde{z}$ are the entire center of $Q^{\prime \prime}$ and $Q^{\prime}$ unless $Q^{\prime \prime}=\mathbb{Z}_{n} \times \mathbb{Z}^{2}$ and $Q^{\prime}=\mathbb{Z} \times \mathbb{Z}^{2}$, respectively. These exceptions occur only when $g=1, p=0$ and $b=0$. If $Q=\mathbb{Z}^{2}$, then $Q^{\prime}$ is nilpotent with center $\mathbb{Z}$ unless $Q^{\prime}=\mathbb{Z} \times \mathbb{Z}^{2}$. The homomorphism $H^{2}(Q ; \mathbb{Z}) \rightarrow H^{2}\left(Q ; \mathbb{Z}_{n}\right)$ given by reduction $\bmod n$ sends a group of the form $Q^{\prime}$ onto the group of the form $0 \rightarrow \mathbb{Z}_{n} \rightarrow Q^{\prime \prime} \rightarrow Q \rightarrow 1$. The presentations of $Q^{\prime}$ and $Q^{\prime \prime}$ show that $\left[Q^{\prime \prime}\right] \in H^{2}\left(Q ; \mathbb{Z}_{n}\right)$ is the $\bmod n$ reduction of $\left[Q^{\prime}\right] \in H^{2}(Q ; \mathbb{Z})$. This establishes Lemma 4.1 .

Now associated with each $\widetilde{Q}$ is an invariant called the euler number of $\widetilde{Q}$ and denoted by $e(\widetilde{Q})$. It is defined to be the real (actually rational) number

$$
e(\widetilde{Q})=-\left(b+\sum_{j=1}^{p} \frac{\beta_{j}}{\alpha_{j}}\right) .
$$


In fact, $|e(\widetilde{Q})|$ is an invariant of the isomorphism class of $\widetilde{Q}$. The number of distinct congruence classes having the same euler number is precisely equal to the order of the torsion in $H^{2}(Q ; \mathbb{Z})$ which is a finite divisor of $\alpha_{1} \cdots \alpha_{p}$, and actually is equal to $\frac{\alpha_{1} \cdots \alpha_{p}}{\operatorname{lcm}\left(\alpha_{1}, \cdots, \alpha_{p}\right)}$. See Theorem 4.5 for more details.

In 4.7, we give a complete solution to the embedding problem when $G=S^{3}$ and $W=\mathbb{R}^{2}$. Crucial to this is the existence of homomorphisms of $\widehat{Q}$ into $S^{1}$ obtained in 4.6. However, it is possible to obtain the results of 4.6 without the elegant cohomological machinery employed in 4.5 and 4.6 by a direct construction involving the euler numbers. We do this in lemmas 4.2, 4.3 and 4.4. While redundant by 4.6, it does offer an alternative concrete approach.

4.2. Lemma. For a given $Q$ with $\chi(Q) \leq 0$, all the possible euler numbers, $e(\widetilde{Q})=$ $-\left(b+\sum_{j=1}^{p} \frac{\beta_{j}}{\alpha_{j}}\right)$, associated to the congruence classes of central extensions of $\mathbb{Z}$ by $Q$ are all the possible integral multiples of $\frac{1}{L}$, where $L=\operatorname{lcm}\left(\alpha_{1}, \cdots, \alpha_{p}\right)$.

Proof. If there are no $\alpha$ 's, then $L=1$; otherwise, we may absorb the integer $b$ into the $\beta$ 's; that is, without loss of generality, we can assume $b=0$ and the $\beta$ 's run through all the possible integers. Put $A=\alpha_{1} \cdots \alpha_{j} \cdots \alpha_{p}$, and

$$
A_{j}=\alpha_{1} \cdots \widehat{\alpha}_{j} \cdots \alpha_{p}=\frac{A}{\alpha_{j}}=\frac{\alpha_{1} \cdots \alpha_{j} \cdots \alpha_{p}}{\alpha_{j}} .
$$

Then it is known that

$$
\operatorname{gcd}\left(A_{1}, \cdots, A_{p}\right) \operatorname{lcm}\left(\alpha_{1}, \cdots, \alpha_{p}\right)=\alpha_{1} \cdots \alpha_{p}=A .
$$

For an integer $y$, the equation

$$
x_{1} A_{1}+x_{2} A_{2}+\cdots+x_{p} A_{p}=y
$$

has integer solutions if and only if $y$ is a multiple of $d=\operatorname{gcd}\left(A_{1}, A_{2}, \cdots, A_{p}\right)$. If we divide the equation by $A=\alpha_{1} \cdots \alpha_{p}$, we get :

$$
\frac{x_{1}}{\alpha_{1}}+\frac{x_{2}}{\alpha_{2}}+\cdots+\frac{x_{p}}{\alpha_{p}}=\frac{y}{A} .
$$

Thus, this equation has integer solutions if and only if $y$ is a multiple of $d$. But $\frac{d}{A}=\frac{1}{L}$, and consequently, we have integer solutions to

$$
\frac{x_{1}}{\alpha_{1}}+\frac{x_{2}}{\alpha_{2}}+\cdots+\frac{x_{p}}{\alpha_{p}}=\frac{1}{L} .
$$

By multiplying any integer to this equality, we see that all the other values of $\sum_{j=1}^{p} x_{j} / \alpha_{j}=e(\widetilde{Q})$ are integral multiples of $\frac{1}{L}$.

We want to understand which $\widehat{Q}$ with a presentation $(\widehat{\mathbf{Q}})$ admits a homomorphism $\widehat{Q} \rightarrow G$. Suppose there is a homomorphism $\theta: \widehat{Q} \rightarrow G$. Let $z$ be a generator of $\mathbb{Z}_{n}$, and let $\tau=\theta(z) \in G$. Then $\widehat{Q}$ must map to the centralizer of $\tau$ in $G$. Suppose the order of $\tau$ is greater than 2. Then $C_{G}(\tau)$ is a circle subgroup $S^{1}$, a maximal torus of $G$.

We can parametrize this $S^{1}$ by $\mathbb{R} / n \mathbb{Z}$ by sending $r \mapsto e^{\frac{2 \pi i}{n} r}$. Then for each $x \in S^{1}$, there exists a unique lift $\widehat{x} \in[0, n)$ with $e^{\frac{2 \pi i}{n} \widehat{x}}=x$. Since $\theta(z)=\tau, \widehat{\tau} \in \mathbb{R}$ will be some integer $s$ between 0 and $n$. That is, $\theta(z)=\tau=\omega^{s}$, where $\omega=e^{\frac{2 \pi i}{n}}$ and $0 \leq s<n$. 
4.3. Lemma. Let $Q$ be any 2-dimensional compact orbifold group with $\chi(Q) \leq 0$. Let $\widehat{Q}$ be a central extension of $\mathbb{Z}_{n}$ by $Q$ with presentation $(\widehat{\mathbf{Q}})$. Then there exists a homomorphism $\hat{\theta}: \widehat{Q} \rightarrow S^{1}$ so that $z \mapsto \omega^{s}, \omega=e^{\frac{2 \pi i}{n}}$, if and only if there exist integers $c, c_{1}, c_{2}, \cdots, c_{p}$ satisfying

$$
s\left(b+\sum_{j=1}^{p} \frac{\beta_{j}}{\alpha_{j}}\right)=n\left(c+\sum_{j=1}^{p} \frac{c_{j}}{\alpha_{j}}\right) .
$$

Then, $\operatorname{Ker}(\hat{\theta}) \cap \mathbb{Z}_{n}=\mathbb{Z}_{k}$, where $k=\operatorname{gcd}(n, s)$, and is generated by $z^{\frac{n}{k}}$. Therefore, $\hat{\theta}$ is one-one on $\mathbb{Z}_{n}$ if and only if $\operatorname{gcd}(n, s)=1$.

Proof. We parametrize $S^{1}$ by $\mathbb{R} / n \mathbb{Z}$ by sending $r \mapsto e^{\frac{2 \pi i}{n} r}$ as before. First assume $\hat{\theta}: \widehat{Q} \rightarrow S^{1}$ exists so that $\hat{\theta}(z)=\tau=\omega^{s}$. We may assume $0 \leq s<n$. Let $\hat{\theta}\left(x_{i}\right)=\lambda_{i}$, $\hat{\theta}\left(y_{i}\right)=\mu_{i}$, and $\hat{\theta}\left(w_{j}\right)=\nu_{j}$. We have unique lifts $\widehat{\lambda_{i}}, \widehat{\mu_{i}}$, and $\widehat{\nu_{j}}$ in $[0, n)$. Since $\hat{\theta}$ is a homomorphism, we have the relations $\nu_{j}^{\alpha_{j}}=\tau^{-\beta_{j}}$, and $\prod_{j=1}^{p} \nu_{j} \prod_{i=1}^{g}\left[\lambda_{i}, \mu_{i}\right]=\tau^{b}$. Note that $\prod_{i=1}^{g}\left[\lambda_{i}, \mu_{i}\right]=1$, and so the last relation becomes $\prod_{j=1}^{p} \nu_{j}=\tau^{b}$. Our lifts satisfy $e^{\frac{2 \pi i}{n} \alpha_{j} \widehat{\nu_{j}}}=\tau^{-\beta_{j}}=e^{\frac{2 \pi i}{n}\left(-\beta_{j}\right) s}$, so that

$$
\alpha_{j} \widehat{\nu_{j}}=-\beta_{j} s+n c_{j}
$$

for some integers $c_{1}, c_{2}, \cdots, c_{p}$. Also from $e^{\frac{2 \pi i}{n}\left(\widehat{\nu_{1}}+\cdots+\widehat{\nu_{p}}\right)}=\tau^{b}=e^{\frac{2 \pi i}{n} s b}$, we have

$$
\widehat{\nu_{1}}+\cdots+\widehat{\nu_{p}}=s b+n(-c)
$$

for some integer $c$. These two equalities yield the equality (4.3.1) easily.

Conversely, if the equality (4.3.1) holds for some integers $c, c_{1}, c_{2}, \cdots, c_{p}$, we shall construct $\hat{\theta}: \widehat{Q} \rightarrow S^{1}$ so that $\hat{\theta}(z)=e^{\frac{2 \pi i}{n} s}=\tau$. For each integer $j$, define

$$
\hat{\theta}\left(w_{j}\right)=\nu_{j},
$$

where $\nu_{j}=e^{\frac{2 \pi i}{n} \widehat{\nu_{j}}}$ and $\widehat{\nu_{j}}=\frac{1}{\alpha_{j}}\left(-\beta_{j} s+n c_{j}\right)$. Then $e^{\frac{2 \pi i}{n} \widehat{\nu_{j}} \alpha_{j}}=e^{\frac{2 \pi i}{n}\left(-\beta_{j} s+n c_{j}\right)}$ $=\tau^{-\beta_{j}}$, so that $\nu_{j}^{\alpha_{j}}=\tau^{-\beta_{j}}$. Define

$$
\widehat{\theta}\left(x_{i}\right)=\lambda_{i}, \quad \widehat{\theta}\left(y_{i}\right)=\mu_{i}
$$

for any choice of $\lambda_{i}, \mu_{i} \in S^{1}$. Then we have $\prod_{i=1}^{g}\left[\lambda_{i}, \mu_{i}\right]=1$ and

$$
\prod_{j=1}^{p} \nu_{j}=e^{\frac{2 \pi i}{n}\left(\widehat{\nu_{1}}+\cdots+\widehat{\nu_{p}}\right)}=e^{\frac{2 \pi i}{n} \sum_{j=1}^{p} \frac{1}{\alpha_{j}}\left(-\beta_{j} s+n c_{j}\right)}=e^{\frac{2 \pi i}{n}(b s-n c)}=e^{\frac{2 \pi i}{n} b s}=\tau^{b} .
$$

Thus, all the relations are satisfied and we obtain a homomorphism $\widehat{\theta}: \widehat{Q} \rightarrow S^{1}$ so that $\widehat{\theta}(z)=e^{\frac{2 \pi i}{n} s}$. Since $k=\operatorname{gcd}(n, s), \theta(z)$ has order $\frac{n}{k}$. Therefore, $\operatorname{Ker}(\hat{\theta}) \cap \mathbb{Z}_{n}=$ $\mathbb{Z}_{k}$, and is generated by $z^{\frac{n}{k}}$.

4.4. Corollary. Let $\widehat{Q}$ be a central extension of $\mathbb{Z}_{n}$ by $Q$ with presentation

$$
\left.\widehat{Q}=\left\langle x_{1}, \cdots, x_{g}, y_{1}, \cdots, y_{g}, z\right| z \text { central, } \quad z^{n}=1, \quad \prod_{i=1}^{g}\left[x_{i}, y_{i}\right]=z^{b}\right\rangle
$$

so that $Q=\widehat{Q} / \mathbb{Z}_{n}$ is a surface group of genus $g$. Then there exists a homomorphism $\hat{\theta}: \widehat{Q} \rightarrow S^{1}$ so that $z \mapsto \omega^{s}, \omega=e^{\frac{2 \pi i}{n}}$, if and only if $s b$ is divisible by $n$. Then $\operatorname{Ker}(\hat{\theta}) \cap \mathbb{Z}_{n}=\mathbb{Z}_{k}$, where $k=\operatorname{gcd}(n, s)$, and is generated by $z^{\frac{n}{k}}$. Therefore, $\hat{\theta}$ is one-one on $\mathbb{Z}_{n}$ if and only if $\widehat{Q} \cong \mathbb{Z}_{n} \times Q$. 
Proof. Since there are no $w$ generators, the condition (4.3.1) reduces to $s b=n c$ for some integer $c$. This implies that $s b$ is divisible by $n$. For $\widehat{\theta}: \widehat{Q} \rightarrow S^{1}$ to be one-one on $\mathbb{Z}_{n}, k=\operatorname{gcd}(n, s)$ must be 1 . Since $n$ is coprime to $s, b$ must be divisible by $n$. But $0 \leq b<n$, so that $b=0$. Now the last relation in the above presentation becomes $\prod_{i=1}^{g}\left[x_{i}, y_{i}\right]=1$ in $S^{1}$. Hence, $\widehat{Q} \cong \mathbb{Z}_{n} \times Q$.

A calculation of the determinant of the $(p+1) \times(p+1)$ relation matrix corresponding to the generators $\hat{w}_{1}, \cdots, \hat{w}_{p}, \hat{z}$ of $\widetilde{Q} /[\widetilde{Q}, \widetilde{Q}]=H_{1}(\widetilde{Q} ; \mathbb{Z})$ yields that the torsion of $H_{1}(\widetilde{Q} ; \mathbb{Z})$ has order $|e(\widetilde{Q})| A$, if $e(\widetilde{Q}) \neq 0$. In particular, if $\alpha_{1}, \cdots, \alpha_{p}$ are pairwise relatively prime, then $L=\alpha_{1} \cdots \alpha_{p}=A$. Consequently, integers $\beta_{1}, \cdots, \beta_{p}$ can be chosen to solve the equation $x_{1} A_{1}+x_{2} A_{2}+\cdots+x_{p} A_{p}=y=d=1$ in 4.2. Using these $\beta_{j}$ 's, we have $\pm[\widetilde{Q}] \in H^{2}(Q ; \mathbb{Z})$, for which $H_{1}( \pm \widetilde{Q} ; \mathbb{Z})$ has trivial torsion. All other congruence classes will have non-trivial torsion in their first homology group, because $\operatorname{lcm}\left(\alpha_{1}, \cdots, \alpha_{p}\right)=A$. Moreover, all the $\beta_{j}$ 's satisfy $\operatorname{gcd}\left(\alpha_{j}, \beta_{j}\right)=1$, for otherwise, the left hand side of the equation for $y$ would contain $\operatorname{gcd}\left(\alpha_{j}, \beta_{j}\right)$ as a factor. This implies that $\widetilde{Q}$ is torsion free, since the resulting Seifert orbifold group is the fundamental group of an aspherical Seifert 3-manifold. (In case $p=0$, i.e., $Q$ is torsion free, then $\widetilde{Q}$ is the fundamental group of a principal $S^{1}$-bundle over a surface of genus $g>0$, and $H_{1}(\widetilde{Q} ; \mathbb{Z})$ is torsion free if and only if $b$ in the presentation for $\widetilde{Q}$ is \pm 1 or 0 .) Therefore, there is a unique congruence class $\widetilde{Q} \in H^{2}(Q ; \mathbb{Z})$ such that $e(\widetilde{Q})=\frac{1}{L}$, whenever the $\alpha_{1}, \cdots, \alpha_{p}$ are pairwise relatively prime. In fact, 4.5 , below, implies that the relatively prime condition is also necessary.

Now we calculate the second cohomology group of $Q$. In the following, we use notation:

$$
\begin{aligned}
& \left(\alpha_{1}, \alpha_{2}, \cdots, \alpha_{k}\right)=\operatorname{gcd}\left(\alpha_{1}, \alpha_{2}, \cdots, \alpha_{k}\right), \\
& {\left[\alpha_{1}, \alpha_{2}, \cdots, \alpha_{k}\right]=\operatorname{lcm}\left(\alpha_{1}, \alpha_{2}, \cdots, \alpha_{k}\right) .}
\end{aligned}
$$

Theorem 4.5. Let $Q$ be a group with a presentation

$$
Q=\left\langle\bar{x}_{1}, \cdots, \bar{x}_{g}, \bar{y}_{1}, \cdots, \bar{y}_{g}, \bar{w}_{1}, \cdots, \bar{w}_{p} \mid \bar{w}_{j}^{\alpha_{j}}=1, \quad \prod_{j=1}^{p} \bar{w}_{j} \prod_{i=1}^{g}\left[\bar{x}_{i}, \bar{y}_{i}\right]=1\right\rangle .
$$

Suppose $\chi(Q) \leq 0$. Then

$$
H^{2}(Q ; \mathbb{Z})=\mathbb{Z}_{d_{1}} \oplus \mathbb{Z}_{d_{2}} \oplus \cdots \mathbb{Z}_{d_{p-1}} \oplus \mathbb{Z},
$$

where the integers $d_{1}, d_{2}, \cdots, d_{p-1}$ are determined by

$$
\begin{aligned}
\left([1], \alpha_{1}\right) & =1, \\
\left(\left[\alpha_{1}\right], \alpha_{2}\right) & =d_{1}, \\
\left(\left[\alpha_{1}, \alpha_{2}\right], \alpha_{3}\right) & =d_{2}, \\
\left(\left[\alpha_{1}, \alpha_{2}, \alpha_{3}\right], \alpha_{4}\right) & =d_{3} \\
\cdots \cdots & \cdots \\
\left(\left[\alpha_{1}, \cdots, \alpha_{p-1}\right], \alpha_{p}\right) & =d_{p-1} .
\end{aligned}
$$

Thus, the order of the torsion in $H^{2}(Q ; \mathbb{Z})$ is equal to $\prod_{j=1}^{p-1} d_{j}=\frac{1}{L} \alpha_{1} \cdots \alpha_{p}$, where $L=\left[\alpha_{1}, \alpha_{2}, \cdots, \alpha_{p}\right]$. 
Let $j: \mathbb{Z} \hookrightarrow \mathbb{R}$ be the inclusion homomorphism. Under the homomorphism

$$
j_{*}: H^{2}(Q ; \mathbb{Z}) \rightarrow H^{2}(Q ; \mathbb{R})
$$

induced by $j$, we have $j_{*}[\widetilde{Q}]=L \cdot e(\widetilde{Q})$.

Proof. Rather than using cocycles, we shall use group presentations. The group $H^{2}(Q ; \mathbb{Z})$ classifies the equivalence classes of central extensions of $\mathbb{Z}$ by $Q$. As stated in 4.1 , when $\chi(Q) \leq 0$, such extensions have presentations of the form

$$
\begin{aligned}
\widetilde{Q}\left(b, \beta_{1}, \cdots, \beta_{p}\right)= & \left\langle\tilde{x}_{1}, \cdots, \tilde{x}_{g}, \tilde{y}_{1}, \cdots, \tilde{y}_{g}, \tilde{w}_{1}, \cdots, \tilde{w}_{p}, \tilde{z}\right| \tilde{z} \text { central, } \\
& \left.\tilde{w}_{j}^{\alpha_{j}}=\tilde{z}^{-\beta_{j}}, \prod_{j=1}^{p} \tilde{w}_{j} \prod_{i=1}^{g}\left[\tilde{x}_{i}, \tilde{y}_{i}\right]=\tilde{z}^{b}\right\rangle
\end{aligned}
$$

and conversely, for any group with a presentation as above, the generator $\tilde{z}$ has infinite order, so that the group is a central extension of $\mathbb{Z}$ by $Q$. Moreover, the $(p+1)$-tuples $\left(b, \beta_{1}, \cdots, \beta_{p}\right)$ can take any integer values. Especially $\widetilde{Q}(0,0, \cdots, 0)=$ $\mathbb{Z} \times Q$, and it represents the 0 element of $H^{2}(Q ; \mathbb{Z})$.

We shall compare a general extension $\widetilde{Q}\left(b, \beta_{1}, \cdots, \beta_{p}\right)$ with $\widetilde{Q}(0,0, \cdots, 0)$. Suppose there exists an isomorphism $\widetilde{Q}\left(b, \beta_{1}, \cdots, \beta_{p}\right) \rightarrow \widetilde{Q}(0,0, \cdots, 0)$ which induces the identity on the kernel $\mathbb{Z}$ and on the quotient $Q$ (i.e., these two groups are congruent). Then it must be of the form:

$$
\begin{aligned}
\tilde{x}_{i} & \mapsto \tilde{z}^{s_{i}} \tilde{x}_{i}, \\
\tilde{y}_{i} & \mapsto \tilde{z}^{t_{i}} \tilde{y}_{i}, \\
\tilde{w}_{j} & \mapsto \tilde{z}^{c_{j}} \tilde{w}_{j}, \\
\tilde{z} & \mapsto z,
\end{aligned}
$$

for some integers $s_{i}, t_{i}$, and $c_{j}(i=1,2, \cdots, g, j=1,2, \cdots, p)$. Applying the isomorphism to the relations $\tilde{w}_{j}^{\alpha_{j}}=\tilde{z}^{-\beta_{j}}$ and $\prod_{j=1}^{p} \tilde{w}_{j} \prod_{i=1}^{g}\left[\tilde{x}_{i}, \tilde{y}_{i}\right]=\tilde{z}^{b}$, we get $\alpha_{j} c_{j}=$ $-\beta_{j}$ for $j=1,2, \cdots, p$ and $\sum_{j=1}^{p} c_{j}=b$. Thus, $\widetilde{Q}\left(b, \beta_{1}, \cdots, \beta_{p}\right) \approx \widetilde{Q}(0,0, \cdots, 0)$ if and only if there exist integers $c_{1}, c_{2}, \cdots, c_{p}$ satisfying

$$
\alpha_{j} c_{j}=-\beta_{j} \quad(j=1,2, \cdots, p) \quad \text { and } \quad \sum_{j=1}^{p} c_{j}=b .
$$

With $c_{1}=0, \cdots, c_{j}=-1, \cdots, c_{p}=0$, for $j=1, \cdots, p$, we get the solution vectors for $\left[b, \beta_{1}, \beta_{2}, \beta_{3}, \cdots, \beta_{p}\right]^{T}$ (T denotes the transpose), in the columns of the $(p+1) \times p$ matrix

$$
A=\left[\begin{array}{cccccc}
-1 & -1 & -1 & -1 & \cdot & -1 \\
\alpha_{1} & 0 & 0 & 0 & \cdot & 0 \\
0 & \alpha_{2} & 0 & 0 & \cdot & 0 \\
0 & 0 & \alpha_{3} & 0 & . & 0 \\
0 & 0 & 0 & \alpha_{4} & \cdot & 0 \\
. & . & . & . & . & . \\
0 & 0 & 0 & 0 & \cdot & \alpha_{p}
\end{array}\right]
$$

The columns of this matrix $A$ generate the extensions which are equivalent to $\widetilde{Q}(0,0, \cdots, 0)$. Consequently, one can view $A$ as a module homomorphism $\mathbb{Z}^{p} \rightarrow$ $\mathbb{Z}^{p+1}$, and then $H^{2}(Q ; \mathbb{Z})$ can be identified with $\operatorname{Coker}(A)=\mathbb{Z}^{p+1} / \operatorname{Im}(A)$. 
In order to find out how the row operations change

$$
\mathbf{b}=\left\{b, \beta_{1}, \beta_{2}, \beta_{3}, \cdots, \beta_{p}\right\}^{T},
$$

we augment the matrix $A$ by the column matrix $\mathbf{b}$, and get $\mathbf{a}(p+1) \times(p+1)$ matrix $[A \mid \mathbf{b}]$. This is not necessary for calculating $H^{2}(Q ; \mathbb{Z})$, but will be used to prove $j_{*}[\widetilde{Q}]=L \cdot e(\widetilde{Q})$. We use elementary row and column operations to diagonalize this matrix $[A \mid \mathbf{b}]$, but avoid any column operation on the last column.

Killing off the $(2,1)$-entry using the first row, and changing the sign of the first two rows, we can change the augmented matrix as follows:

$$
[A \mid \mathbf{b}] \longmapsto A^{\prime}=\left[\begin{array}{cccccc|c}
1 & 1 & 1 & 1 & \cdot & 1 & -b \\
0 & \alpha_{1} & \alpha_{1} & \alpha_{1} & . & \alpha_{1} & -\left(\alpha_{1} b+\beta_{1}\right) \\
0 & \alpha_{2} & 0 & 0 & . & 0 & \beta_{2} \\
0 & 0 & \alpha_{3} & 0 & . & 0 & \beta_{3} \\
0 & 0 & 0 & \alpha_{4} & . & 0 & \beta_{4} \\
\cdot & \cdot & \cdot & \cdot & . & \cdot & \cdot \\
0 & 0 & 0 & 0 & \cdot & \alpha_{p} & \beta_{p}
\end{array}\right]
$$

Using elementary column operations, we can kill off the 1's on the first row. Also noting that $\alpha_{1} b+\beta_{1}=\alpha_{1}\left(b+\frac{\beta_{1}}{\alpha_{1}}\right)$, we get

$$
A^{\prime \prime}=\left[\begin{array}{cccccc|c}
1 & 0 & 0 & 0 & \cdot & 0 & * \\
0 & \alpha_{1} & \alpha_{1} & \alpha_{1} & \cdot & \alpha_{1} & -\alpha_{1}\left(b+\frac{\beta_{1}}{\alpha_{1}}\right) \\
0 & \alpha_{2} & 0 & 0 & \cdot & 0 & \beta_{2} \\
0 & 0 & \alpha_{3} & 0 & \cdot & 0 & \beta_{3} \\
0 & 0 & 0 & \alpha_{4} & \cdot & 0 & \beta_{4} \\
\cdot & \cdot & \cdot & \cdot & \cdot & \cdot & \cdot \\
0 & 0 & 0 & 0 & \cdot & \alpha_{p} & \beta_{p}
\end{array}\right]
$$

Consider the second and third rows of this new matrix with the first column removed:

$$
\left[\begin{array}{cccccc|c}
\alpha_{1} & \alpha_{1} & \alpha_{1} & \cdot & \cdot & \alpha_{1} & -\alpha_{1}\left(b+\frac{\beta_{1}}{\alpha_{1}}\right) \\
\alpha_{2} & 0 & 0 & \cdot & \cdot & 0 & \beta_{2}
\end{array}\right]
$$

Lemma. Applying the Euclidean algorithm to the $(1,1)$ and $(2,1)$ entries, and corresponding elementary row operations, we can change this matrix to

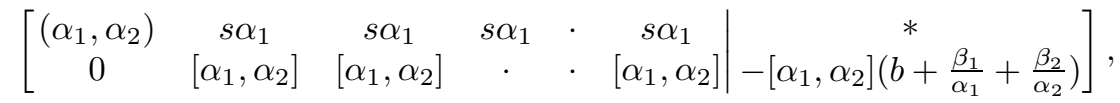

where $(a, b)=\operatorname{gcd}(a, b),[a, b]=\operatorname{lcm}(a, b)$ and $s \in \mathbb{Z}$.

Proof. Let $\left(\alpha_{1}, \alpha_{2}\right)=d, \alpha_{1}=d a_{1}, \alpha_{2}=d a_{2}$. Then $\left[\alpha_{1}, \alpha_{2}\right]=\alpha_{1} a_{2}=a_{1} \alpha_{2}$. Let $s, t$ be integers such that $s \alpha_{1}+t \alpha_{2}=d$. Then

$$
\left[\begin{array}{cc}
s & t \\
a_{2} & -a_{1}
\end{array}\right]\left[\begin{array}{cccccc|c}
\alpha_{1} & \alpha_{1} & \alpha_{1} & \cdot & \cdot & \alpha_{1} & -\alpha_{1}\left(b+\frac{\beta_{1}}{\alpha_{1}}\right) \\
\alpha_{2} & 0 & 0 & \cdot & \cdot & 0 & \beta_{2}
\end{array}\right]
$$

gives the matrix in the statement. Since

$$
\left[\begin{array}{cc}
s & t \\
a_{2} & -a_{1}
\end{array}\right]
$$

has determinant -1 , it is a product of elementary integral matrices. This finishes the proof of the lemma. 
Now, replace the second and third row of $A^{\prime \prime}$ by the above. The entries $s \alpha_{1}$ are divisible by $\left(\alpha_{1}, \alpha_{2}\right)$. Therefore, the entries right to $\left(\alpha_{1}, \alpha_{2}\right)$ of the unaugmented part can be killed off by elementary column operations. Thus, we get

$$
\left[\begin{array}{cccccc|c}
1 & 0 & 0 & 0 & . & 0 & * \\
0 & \left(\alpha_{1}, \alpha_{2}\right) & 0 & 0 & . & 0 & * \\
0 & 0 & {\left[\alpha_{1}, \alpha_{2}\right]} & {\left[\alpha_{1}, \alpha_{2}\right]} & \cdot & {\left[\alpha_{1}, \alpha_{2}\right]} & -\left[\alpha_{1}, \alpha_{2}\right]\left(b+\frac{\beta_{1}}{\alpha_{1}}+\frac{\beta_{2}}{\alpha_{2}}\right) \\
0 & 0 & \alpha_{3} & 0 & . & 0 & \beta_{3} \\
0 & 0 & 0 & \alpha_{4} & \cdot & 0 & \beta_{4} \\
. & . & . & . & . & . & \cdot \\
0 & 0 & 0 & 0 & . & \alpha_{p} & \beta_{p}
\end{array}\right]
$$

We can apply the lemma to the fourth and fifth rows. Note also that

$$
\left[\left[\alpha_{1}, \cdots, \alpha_{j-1}\right], \alpha_{j}\right]=\left[\alpha_{1}, \cdots, \alpha_{j-1}, \alpha_{j}\right],
$$

and continue this process.

Consequently, there exist matrices $X, Y \in \mathrm{GL}(p+1, \mathbb{Z})$, with the last column of $\mathrm{Y}$ being $[0,0, \cdots, 0,1]^{T}$ since we never used any column operation on the last column, such that

$$
X^{-1}[A \mid \mathbf{b}] Y=\left[\begin{array}{cccccc|c}
1 & 0 & 0 & 0 & \cdot & 0 & * \\
0 & d_{1} & 0 & 0 & . & 0 & * \\
0 & 0 & d_{2} & 0 & . & 0 & * \\
0 & 0 & 0 & d_{3} & . & 0 & * \\
. & . & . & . & . & \cdot & \cdot \\
0 & 0 & 0 & 0 & \cdot & d_{p-1} & * \\
0 & 0 & 0 & 0 & . & 0 & B
\end{array}\right]
$$

where

$$
\begin{aligned}
\left([1], \alpha_{1}\right) & =1, \\
\left(\left[\alpha_{1}\right], \alpha_{2}\right) & =d_{1}, \\
\left(\left[\alpha_{1}, \alpha_{2}\right], \alpha_{3}\right) & =d_{2}, \\
\left(\left[\alpha_{1}, \alpha_{2}, \alpha_{3}\right], \alpha_{4}\right) & =d_{3}, \\
\cdots \cdots & \\
\left(\left[\alpha_{1}, \cdots, \alpha_{p-1}\right], \alpha_{p}\right) & =d_{p-1}, \\
-\left[\alpha_{1}, \cdots, \alpha_{p}\right]\left(b+\sum_{j=1}^{p} \frac{\beta_{j}}{\alpha_{j}}\right) & =B .
\end{aligned}
$$

We have proved that

$$
H^{2}(Q ; \mathbb{Z})=\mathbb{Z}_{d_{1}} \oplus \mathbb{Z}_{d_{2}} \oplus \cdots \mathbb{Z}_{d_{p-1}} \oplus \mathbb{Z}
$$

The homomorphism

$$
j_{*}: H^{2}(Q ; \mathbb{Z}) \rightarrow H^{2}(Q ; \mathbb{R})
$$

induced by the inclusion homomorphism $j: \mathbb{Z} \hookrightarrow \mathbb{R}$ is just $\otimes \mathbb{R}$. More precisely, the homomorphism $A: \mathbb{Z}^{p} \rightarrow \mathbb{Z}^{p+1}$ extends uniquely to $\tilde{A}: \mathbb{R}^{p} \rightarrow \mathbb{R}^{p+1}$. Then $H^{2}(Q ; \mathbb{R})=\operatorname{Coker}(\tilde{A})$. Thus,

$$
j_{*}: H^{2}(Q ; \mathbb{Z})=\mathbb{Z}^{p+1} / \operatorname{Im}(A) \stackrel{\otimes \mathbb{R}}{\longrightarrow} \mathbb{R}^{p+1} / \operatorname{Im}(\tilde{A})=H^{2}(Q ; \mathbb{R}) .
$$


The procedure of diagonalizing the matrix $[A \mid \mathbf{b}]$ shows that the column matrix $\mathbf{b}=\left\{b, \beta_{1}, \beta_{2}, \beta_{3}, \cdots, \beta_{p}\right\}^{T}$ changes to $\{*, *, *, \cdots, *, B\}^{T}$. Thus, under

$$
\begin{aligned}
\mathbb{Z}^{p+1} & \rightarrow \mathbb{Z}_{d_{1}} \oplus \mathbb{Z}_{d_{2}} \oplus \cdots \oplus \mathbb{Z}_{d_{p-1}} \oplus \mathbb{Z} \\
\mathbf{b} & \mapsto(*, \quad *, \cdots, \quad *, B)
\end{aligned}
$$

$j_{*}$ is just picking out the last entry $B$. Consequently,

$$
\begin{aligned}
j_{*}\left(\widetilde{Q}\left(b, \beta_{1}, \cdots, \beta_{p}\right)\right) & =B=-\left[\alpha_{1}, \cdots, \alpha_{p-1}, \alpha_{p}\right]\left(b+\sum_{j=1}^{p} \frac{\beta_{j}}{\alpha_{j}}\right) \\
& =L \cdot e\left(\widetilde{Q}\left(b, \beta_{1}, \cdots, \beta_{p}\right)\right)
\end{aligned}
$$

where $L=\left[\alpha_{1}, \alpha_{2}, \cdots, \alpha_{p}\right]$, since $e\left(\widetilde{Q}\left(b, \beta_{1}, \cdots, \beta_{p}\right)\right)=-\left(b+\sum_{j=1}^{p} \frac{\beta_{j}}{\alpha_{j}}\right)$.

To prove $\prod_{j=1}^{p-1} d_{j}=\frac{1}{L} \alpha_{1} \cdots \alpha_{p}$, where $L=\left[\alpha_{1}, \alpha_{2}, \cdots, \alpha_{p}\right]$, we use the fact $x \cdot y=(x, y) \cdot[x, y]$ repeatedly. From the definitions of integers $d_{1}, d_{2}, \cdots, d_{p-1}$, we get

$$
\begin{aligned}
{[1] \cdot \alpha_{1} } & =1 \cdot\left[\alpha_{1}\right], \\
{\left[\alpha_{1}\right] \cdot \alpha_{2} } & =d_{1} \cdot\left[\alpha_{1}, \alpha_{2}\right], \\
{\left[\alpha_{1}, \alpha_{2}\right] \cdot } & \alpha_{3}=d_{2} \cdot\left[\alpha_{1}, \alpha_{2}, \alpha_{3}\right], \\
{\left[\alpha_{1}, \alpha_{2}, \alpha_{3}\right] \cdot \alpha_{4} } & =d_{3} \cdot\left[\alpha_{1}, \alpha_{2}, \alpha_{3}, \alpha_{4}\right], \\
\cdots & \cdots \\
{\left[\alpha_{1}, \cdots, \alpha_{p-1}\right] \cdot } & \alpha_{p}=d_{p-1} \cdot\left[\alpha_{1}, \cdots, \alpha_{p}\right] .
\end{aligned}
$$

By multiplying each side, we get

$$
\alpha_{1} \cdot \alpha_{2} \cdots \alpha_{p}=d_{1} \cdot d_{2} \cdots d_{p-1} \cdot\left[\alpha_{1}, \alpha_{2}, \cdots, \alpha_{p}\right]
$$

Thus, we have shown that the order of the torsion of $H^{2}(Q ; \mathbb{Z})$ is

$$
\alpha_{1} \cdots \alpha_{p} / \operatorname{lcm}\left(\alpha_{1}, \cdots, \alpha_{p}\right) .
$$

Observe that $e: \widetilde{Q} \mapsto e(\widetilde{Q})$ also defines a homomorphism $e: H^{2}(Q ; \mathbb{Z}) \rightarrow \mathbb{R} \cong$ $H^{2}(Q ; \mathbb{R})$, and $\left|e^{-1}(e(\widetilde{Q}))\right|=d_{1} \cdots d_{p-1}$ only depends upon $Q$.

Remark. We may obtain a calculation for all the cohomology $H^{*}(Q ; \mathbb{Z})$ by more geometric means, but with a little less precision at $H^{2}(Q ; \mathbb{Z})$, as follows. We may construct $K(Q, 1)$ by taking $W=\mathbb{R}^{2}$ or $\mathbf{H}^{2}$ and letting $Q$ act isometrically. On $Q \backslash W$, remove the interior of a 2 -disk $D_{i}$ about each of the elliptic points $x_{i}$. Replace each $D_{i}$ by attaching $K\left(\mathbb{Z}_{\alpha_{i}}, 1\right)$ by a homeomorphism of the 1 -skeleton. (We think of $K\left(\mathbb{Z}_{\alpha_{i}}, 1\right)$ as an infinite dimensional lens space obtained from the quotient of the infinite sphere $S^{\infty}=\bigcup_{\ell=1}^{\infty} S^{\ell}$ by a free action of $\mathbb{Z}_{\alpha_{i}}$.) Call this complex $B$. Passing to the universal covering of $B$, we get $\mathbb{R}^{2}$ or $\mathbf{H}^{2}$ with an infinite number of open disks replaced by the contractible $S^{\infty}$ 's. The universal covering $\widetilde{B}$ is contractible, and $Q$ acts freely as covering transformations yielding $Q \backslash \widetilde{B}=B=K(Q, 1)$. Now, if we remove an open 2-disk from $B$ whose closure is disjoint from the attached $K\left(\mathbb{Z}_{\alpha_{i}}, 1\right)$ 's, we get a complex $B^{\prime}$, which deformation 
retracts to a wedge $\left(\bigvee_{i=1}^{p} K\left(\mathbb{Z}_{\alpha_{i}}, 1\right)\right) \vee\left(\bigvee_{j=1}^{2 g} S_{j=1}^{1}\right)$. Then

$$
H^{j}\left(B^{\prime} ; \mathbb{Z}\right)= \begin{cases}\mathbb{Z}, & j=0, \\ \mathbb{Z}^{2 g}, & j=1, \\ 0, & j \text { odd }, j>1, \\ \mathbb{Z}_{\alpha_{1}} \oplus \cdots \oplus \mathbb{Z}_{\alpha_{p}}, & j \text { even, } j>0 .\end{cases}
$$

Moreover, $H^{j}\left(B, B^{\prime} ; \mathbb{Z}\right)=0, j \neq 2$, since the difference is an open $2-$ cell. Then the cohomology exact sequence of the pair $\left(B, B^{\prime}\right)$ yields

$$
H^{j}(B ; \mathbb{Z}) \cong H^{j}\left(B^{\prime} ; \mathbb{Z}\right), \text { for } j \neq 2,
$$

and for dimension 2, we have the exact sequence

$$
0 \rightarrow \mathbb{Z} \rightarrow H^{2}(B ; \mathbb{Z}) \rightarrow \mathbb{Z}_{\alpha_{1}} \oplus \cdots \oplus \mathbb{Z}_{\alpha_{p}} \rightarrow 0
$$

This is immediate if $g=0$; a more careful analysis is needed using the cell structure of $B$ if $g>0$. In particular, $H^{3}(Q ; \mathbb{Z})=H^{3}(B ; \mathbb{Z})=0$. This fact will be used in 4.6. Notice this also yields the ring structure of $H^{*}(Q ; \mathbb{Z})$, since we know it for $H^{*}\left(B^{\prime} ; \mathbb{Z}\right)$. To evaluate $a \cup b$, with $a \in H^{2}(Q ; \mathbb{Z})$ and $b \in H^{j}(Q ; \mathbb{Z})$, assume that $a$ hits a generator of $\mathbb{Z}_{\alpha_{i}}$ for each $i$ under the homomorphism $H^{2}(B ; \mathbb{Z}) \rightarrow$ $\mathbb{Z}_{\alpha_{1}}+\cdots+\mathbb{Z}_{\alpha_{p}}$. (Such an $a$ always exists.) Then $b \rightarrow a \cup b$ induces an isomorphism $H^{j}(B ; \mathbb{Z}) \rightarrow H^{j+2}(B ; \mathbb{Z}), j>2$.

For a different and more general approach the reader is referred to the cohomology long exact sequence in [2, p.262].

Definition. Let $\widehat{Q}$ be a central extension of $\mathbb{Z}_{n}$ by $Q, \chi(Q) \leq 0$, with presentation

$$
\begin{gathered}
\widehat{Q}=\left\langle x_{1}, \cdots, x_{g}, y_{1}, \cdots, y_{g}, w_{1}, \cdots, w_{p}, z\right| z \text { central, } \quad z^{n}=1 w_{j}^{\alpha_{j}}=z^{-\beta_{j}}, \\
\left.\prod_{j=1}^{p} w_{j} \prod_{i=1}^{g}\left[x_{i}, y_{i}\right]=z^{b}\right\rangle .
\end{gathered}
$$

An integer $\mathcal{E}(\widehat{Q})$ between 0 and $n-1$ is defined by :

$$
\mathcal{E}(\widehat{Q})=-\left[\alpha_{1}, \alpha_{2}, \cdots, \alpha_{p}\right]\left(b+\sum_{j=1}^{p} \frac{\beta_{j}}{\alpha_{j}}\right) \bmod n .
$$

4.6. Corollary. Let $Q$ be a group with presentation $(\mathbf{Q})$ and $\chi(Q) \leq 0$; and let $\widehat{Q}$ be a central extension of $\mathbb{Z}_{n}$ by $Q$ with presentation $(\widehat{\mathbf{Q}})$.

1. Let $Q^{\prime}$ be any central extension of $\mathbb{Z}$ by $Q$ whose $\bmod n$ reduction is $\widehat{Q}$. Then $L \cdot e\left(Q^{\prime}\right) \equiv \mathcal{E}(\widehat{Q}) \bmod n$.

2. There is a natural injective homomorphism $\widehat{Q} \rightarrow S^{1} \times Q$ extending $\mathbb{Z}_{n} \hookrightarrow S^{1}$ if and only if $\mathcal{E}(\widehat{Q})=0$.

Proof. (1) Observe that there are many $Q^{\prime} \in H^{2}(Q ; \mathbb{Z})$ which naturally map down to $\widehat{Q}$. We use the result in Lemma 3.4. By the Remark after $4.5, H^{3}(Q ; \mathbb{Z})=0$. 
The commutative diagram with exact rows in the proof of 3.4 becomes

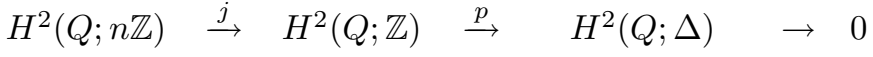

$$
\begin{aligned}
& \| \quad \downarrow j_{*} \quad \downarrow \bar{j}_{*} \\
& H^{2}(Q ; n \mathbb{Z}) \stackrel{j^{\prime}}{\longrightarrow} H^{2}(Q ; \mathbb{R}) \stackrel{p^{\prime}}{\longrightarrow} H^{2}(Q ; \mathrm{SO}(2)) \quad \rightarrow \quad 0
\end{aligned}
$$

The exactness implies that there always exists an extension $Q^{\prime} \in H^{2}(Q ; \mathbb{Z})$ of $\mathbb{Z}$ by $Q$ such that $p\left[Q^{\prime}\right]=[\widehat{Q}]$. (In fact, an obvious one is just deleting the relation $z^{n}=1$ from the presentation of $\widehat{Q}$ ). Therefore, Condition $(\mathbf{C 1})$ in 3.6 automatically holds. For $\widehat{Q}, \mathcal{E}(\widehat{Q})$ was defined by $L \cdot e\left(Q^{\prime}\right) \bmod n=j_{*}\left[Q^{\prime}\right] \bmod n$ by Theorem 4.5 , where $Q^{\prime} \in H^{2}(Q ; \mathbb{Z})$ is such that $p\left[Q^{\prime}\right]=\widehat{Q}$. To show that $j_{*}\left[Q^{\prime}\right] \bmod n$ is independent of the choice of $Q^{\prime}$, let $Q_{1}^{\prime}, Q_{2}^{\prime}$ be elements of $H^{2}(Q ; \mathbb{Z})$ such that $p\left[Q_{i}^{\prime}\right]=\widehat{Q}$ for $i=1,2$. By diagram chasing, $j_{*}\left[Q_{1}^{\prime}\right]-j_{*}\left[Q_{2}^{\prime}\right]$ lies in the image of $j^{\prime}$. But $\operatorname{Im}\left(j^{\prime}\right)=n \cdot \operatorname{Im}\left(j_{*}\right)=n \mathbb{Z}$ because $j_{*}=1 \otimes \mathbb{R}$. (This can be seen also from Lemma 4.2. It implies that there exists an element $\left[Q^{\prime \prime}\right] \in H^{2}(Q ; \mathbb{Z})$ so that $j_{*}\left[Q^{\prime \prime}\right]=L \cdot e\left(Q^{\prime \prime}\right)=1$.) Therefore, $j_{*}\left[Q_{1}^{\prime}\right]=j_{*}\left[Q_{2}^{\prime}\right] \bmod n$. This proves $(1)$.

(2) There is a natural injective homomorphism $\widehat{Q} \rightarrow S^{1} \times Q$ extending $\mathbb{Z}_{n} \hookrightarrow S^{1}$ if and only if $\bar{j}_{*}[\widehat{Q}]=0 \in H^{2}(Q ; \mathrm{SO}(2))$. Now, for $\bar{j}_{*}[\widehat{Q}]=0$, it is necessary and sufficient that $j_{*}[\widetilde{Q}]$ lies in the image of $H^{2}(Q ; n \mathbb{Z})$ so that $j_{*}[\widetilde{Q}] \equiv 0 \bmod n$, which is equivalent to saying that $\mathcal{E}(\widehat{Q})=0$. This proves the corollary.

Combining 3.6 and 4.6, we get a complete statement of conditions for embeddability of an extension of a spherical space-form group $\Delta$ by a cocompact orbifold group $Q \subset \operatorname{Isom}_{0}(W)$ for $W=\mathbb{R}^{2}$ or $\mathbf{H}^{2}$. Let

$$
Q=\left\langle\bar{x}_{1}, \cdots, \bar{x}_{g}, \bar{y}_{1}, \cdots, \bar{y}_{g}, \bar{w}_{1}, \cdots, \bar{w}_{p} \mid \bar{w}_{j}^{\alpha_{j}}=1, \quad \prod_{j=1}^{p} \bar{w}_{j} \prod_{i=1}^{g}\left[\bar{x}_{i}, \bar{y}_{i}\right]=1\right\rangle
$$

be a discrete subgroup of $\operatorname{Isom}_{0}(W)$ for $W=\mathbb{R}^{2}$, or $\mathbf{H}^{2}$. Then Condition (C1) in 3.6 is automatic (see the proof of 4.6), and (C2) can be expressed in terms of $\mathcal{E}\left(Q^{\prime}\right)$. We restate these two conditions for extensions of a cyclic group or binary polyhedral group by $Q$ :

For an extension $Q^{\prime}$ of a non-cyclic binary polyhedral group $\mathbf{B}^{*}$ by $Q$,

\section{Condition (B) :}

1. If $\mathbf{B}^{*}=\mathbf{D}_{8}^{*}, Q^{\prime}$ is either a product $\mathbf{D}_{8}^{*} \times Q$, a diagonal subgroup of $\mathbf{D}_{16}^{*} \times Q$ of index 2 , a diagonal subgroup of $\mathbf{T}^{*} \times Q$ of index 3 , or a diagonal subgroup of $\mathbf{O}^{*} \times Q$ of index 6 .

2. If $\mathbf{B}^{*}=\mathbf{D}_{4 k}^{*}(k>2)$ or $\mathbf{T}^{*}$, then $Q^{\prime}$ is either $\mathbf{B}^{*} \times Q$ or a diagonal subgroup of $\mathbf{D}_{8 k}^{*} \times Q$ or $\mathbf{O}^{*} \times Q$ of index 2 , respectively.

3. If $\mathbf{B}^{*}=\mathbf{O}^{*}$ or $\mathbf{I}^{*}$, then $Q^{\prime}=\mathbf{B}^{*} \times Q$.

For an extension $Q^{\prime}$ of a cyclic group $\mathbb{Z}_{n}$ by $Q$,

\section{Conditions (C1) and (C2).}

(C1) There exists a crossed homomorphism $\tilde{i}: Q^{\prime} \rightarrow \mathrm{M}\left(W, S^{3} \rtimes_{\mathbb{Z}_{2}} \mathbb{Z}_{4}\right)$ extending $\mathbb{Z}_{n} \hookrightarrow \mathrm{SO}(2)$. If $n>2$, then this reduces to: there exists a crossed homomorphism $\tilde{i}: Q^{\prime} \rightarrow \mathrm{M}\left(W, \mathrm{SO}(2) \rtimes_{\mathbb{Z}_{2}} \mathbb{Z}_{4}\right)$ extending $\mathbb{Z}_{n} \hookrightarrow \mathrm{SO}(2)$. If $\Delta$ is central and $n>2$, there is no condition. 
(C2) There exists a homomorphism $Q^{\prime} \rightarrow S^{3}$ extending $\mathbb{Z}_{n} \hookrightarrow \operatorname{SO}(2) \subset S^{3}$. If $n>2$, then this reduces to: there exists a homomorphism $Q^{\prime} \rightarrow \mathrm{SO}(2) \rtimes_{\mathbb{Z}_{2}} \mathbb{Z}_{4}$ extending $\mathbb{Z}_{n} \hookrightarrow \mathrm{SO}(2)$. If $\mathbb{Z}_{n}$ is central and $n>2$, then this is equivalent to $\mathcal{E}\left(Q^{\prime}\right)=0$.

4.7. Theorem. Let $i=i_{\ell} \times i_{r}: \Delta \hookrightarrow \ell(G) \times r(G)$ be a spherical space-form group; let $Q \subset \operatorname{Isom}_{0}(W)$, where $W=\mathbb{R}^{2}$ or $\mathbf{H}^{2}$, be a group with presentation $(\mathbf{Q})$ with $\chi(Q) \leq 0$; and let $1 \rightarrow \Delta \rightarrow \Pi \rightarrow Q \rightarrow 1$ be exact. Then there exists an injective homomorphism $\theta: \Pi \rightarrow \ell(G) \times[\mathrm{M}(W, G) \rtimes \operatorname{Isom}(W)]$ which makes the diagram (15) in 3.6 commutative if and only if $\Pi$ satisfies the following:

1. If $\Delta_{\ell}$ is cyclic and $\Delta_{r}$ is not cyclic: $\Pi / K_{\ell}$ satisfies $(\mathbf{C 2})$, and $\Pi / K_{r}$ satisfies (B).

2. If $\Delta_{r}$ is cyclic and $\Delta_{\ell}$ is not cyclic: $\Pi / K_{\ell}$ satisfies $(\mathbf{B})$, and $\Pi / K_{r}$ satisfies (C1).

3. If $\Delta_{r}$ and $\Delta_{\ell}$ are both cyclic: $\Pi / K_{\ell}$ satisfies $(\mathbf{C 2})$, and $\Pi / K_{r}$ satisfies (C1).

In 2, if, furthermore, $\Pi / K_{r}$ satisfies $(\mathbf{C 2})$, then $\Pi$ embeds into $\operatorname{Isom}_{0}(G \times W)$.

Remark. (1) Since cocompact and discrete $Q$ in $\operatorname{TOP}(W)$ can be conjugated into $\operatorname{Isom}(W)$, we obtain the same result as above if we replace $\operatorname{Isom}(W)$ with $\operatorname{TOP}(W)$.

(2) It is possible, for $\chi(Q) \leq 0$, to determine when $1 \rightarrow \mathbb{Z}_{n} \rightarrow \widehat{Q} \rightarrow Q \rightarrow 1$ acts freely on $G \times W$ when $\mathbb{Z}_{n}$ embeds in $r(G)$. From the way we have defined our action on $S^{1} \times W$, we see that it actually was $\widehat{Q}$ acting on $\mathbb{R}^{1} / n \mathbb{Z} \times W$. But this is the same as the action of $\widetilde{Q}$ on $\mathbb{R} \times W$. Therefore, as a space $\widehat{Q} \backslash\left(S^{1} \times W\right)$, we see it as $\widetilde{Q} \backslash\left(\mathbb{R}^{1} \times W\right)=\widehat{Q} \backslash\left(\mathbb{R}^{1} / n \mathbb{Z} \times W\right)$, which projects to $Q \backslash W$. For the action $\left(\widehat{Q}, \mathbb{R}^{1} / n \mathbb{Z} \times W\right)$ to be free, it is necessary and sufficient that the action of $\left(\widetilde{Q}, \mathbb{R}^{1} \times W\right)$ be free, because the action of $n \mathbb{Z} \subset \widetilde{Q}$ on $\mathbb{R}^{1} \times W$ is already free. The necessary and sufficient condition that $\left(\widetilde{Q}, \mathbb{R}^{1} \times W\right)$ be free is that $\operatorname{gcd}\left(\alpha_{j}, \beta_{j}\right)=1$ for all $j$. (This is also equivalent to saying that $\widetilde{Q}$ as a group is torsion free.)

We shall give a few instructive examples of embedding $\Pi$ into $\tilde{\mathcal{U}}$.

4.8. Example. Consider a group $\Pi$ with presentation

$$
\left.\Pi=\langle x, y, z| z \text { central, } \quad z^{12}=1,[x, y]=z^{8}\right\rangle .
$$

Then $\Pi$ is a central extension of $\Delta=\mathbb{Z}_{12}$ by $\mathbb{Z}^{2}$. Suppose $\mathbb{Z}^{2}=\langle x, y\rangle$ acts on $\mathbb{R}^{2}$ via the standard translations $\rho(x)=t_{1}, \rho(y)=t_{2}$. We shall study the embeddability of $\Pi$ into $\widetilde{\mathcal{U}}$, which acts freely on $G$, for all possible representations $i=i_{\ell} \times i_{r}: \Delta=\mathbb{Z}_{12} \subset \ell(G) \times r(G)$ If $\Pi$ embeds, it will act on $G \times \mathbb{R}^{2}$ properly and freely.

Consider all possible embeddings of $\mathbb{Z}_{12}$ into $\ell(G) \times r(G)$, which acts freely on $G$. Let $z$ be a fixed generator of $\mathbb{Z}_{12}$. In 3.2(1), integers $r$ coprime to $n=12$ are $1,5,7,11$. Let us denote $\mathcal{R}\left(\frac{1}{12}\right)$ by $\omega$. For example, for $r=5$, we get $\frac{1+r}{2 n}=\frac{3}{12}$ and $\frac{1-r}{2 n}=\frac{-2}{12}$. Therefore, $z \mapsto\left(\omega^{3}, \omega^{-2}\right) \in \ell(G) \times r(G)$. The following are all possible embeddings of $\mathbb{Z}_{12}$ into $\ell(G) \times r(G)$ so that $\mathbb{Z}_{12}$ acts freely on $G$. We write the 
image of the generator $z \mapsto\left(\omega^{s}, \omega^{s^{\prime}}\right)$ :

$$
\begin{array}{ll}
r=1: & z \mapsto\left(\omega^{1}, \omega^{0}\right), \\
r=5: & z \mapsto\left(\omega^{3}, \omega^{-2}\right), \\
r=7: & z \mapsto\left(\omega^{4}, \omega^{-3}\right), \\
r=11: & z \mapsto\left(\omega^{6}, \omega^{-5}\right) .
\end{array}
$$

The other representations come from conjugations by elements of $\mathrm{SO}(4)$ or multiplying by $\left(\omega^{6}, \omega^{6}\right)=(-1,-1)$. In fact, among these 4 cases, two pairs $(r=1$ and $r=11)$ and $(r=5$ and $r=7)$ give conjugate representations in $\mathrm{O}(4)$. However, for the purpose of the Seifert construction, we need to treat them differently. We apply Theorem 4.7 , case 3 .

Since $\Delta_{\ell}$ is cyclic and central in $\Pi / K_{\ell}$, the condition $(\mathbf{C 2})$ requires that $\mathcal{E}\left(\Pi / K_{\ell}\right)$ $=0$. For $s=1,3,4$, and $6, \Delta_{\ell}=\mathbb{Z}_{12}, \mathbb{Z}_{4}, \mathbb{Z}_{3}$ and $\mathbb{Z}_{2}$. Then $\mathcal{E}\left(\Pi / K_{\ell}\right)=8 \bmod$ $12,4,3,2$. Only two cases $s=3$ and 6 yield $\mathcal{E}\left(\Pi / K_{\ell}\right)=0$. (This follows also from 4.4.) Corresponding to these, we have $s^{\prime}=-2,-5$. Since (C2) is the only requirement, there exists a Seifert construction for each of these two cases $(s=3,6)$. For the other two cases $(s=1,4)$, there are no Seifert constructions.

We proceed to obtain explicit embeddings of $\Pi$ into $\tilde{\mathcal{U}}$ as follows. We work out the case when $i=i_{\ell} \times i_{r}: \Delta \subset \ell(G) \times r(G)$ is given by $z \mapsto\left(\omega^{3}, \omega^{-2}\right)$. It is clear, from $z \mapsto\left(\omega^{3}, \omega^{-2}\right) \in \ell(G) \times r(G)$, that

$$
\begin{array}{ll}
K_{\ell} \cong \mathbb{Z}_{3}=\left\langle z^{4}\right\rangle, & K_{r} \cong \mathbb{Z}_{2}=\left\langle z^{6}\right\rangle, \\
\Delta_{\ell} \cong \mathbb{Z}_{4}=\left\langle\omega^{3}\right\rangle, & \Delta_{r} \cong \mathbb{Z}_{6}=\left\langle\omega^{-2}\right\rangle .
\end{array}
$$

Also note that

$$
\begin{array}{ll}
\left.\Pi / K_{\ell}=\langle x, y, \bar{z}| \bar{z} \text { central, } \bar{z}^{4}=1,[x, y]=1\right\rangle & \left(\text { extension of } \mathbb{Z}_{4} \text { by } \mathbb{Z}^{2}\right), \\
\left.\Pi / K_{r}=\langle x, y, \bar{z}| \bar{z} \text { central, } \bar{z}^{6}=1,[x, y]=\bar{z}^{2}\right\rangle & \left(\text { extension of } \mathbb{Z}_{6} \text { by } \mathbb{Z}^{2}\right),
\end{array}
$$

since $8 \equiv 0 \bmod 4$ and $8 \equiv 2 \bmod 6$.

First, we define a homomorphism $\Pi \rightarrow \Pi / K_{\ell} \rightarrow \ell(G)$ by $z \mapsto \omega^{s}, x \mapsto 1$ and $y \mapsto 1$. This is possible because the condition $(\mathbf{C 2})$ is met. In fact, $\Pi / K_{\ell}=$ $\Pi / \mathbb{Z}_{4}=\mathbb{Z}_{4} \times \mathbb{Z}^{2}$ by 4.4 .

Next, we shall find an explicit homomorphism $\Pi \rightarrow \Pi / K_{r} \rightarrow \mathrm{M}\left(\mathbb{R}^{2}, S^{1}\right) \rtimes$ $\operatorname{TOP}\left(\mathbb{R}^{2}\right)$ so that $\Delta$ is mapped via $z \mapsto\left(\omega^{s^{\prime}}, 1\right) \in \mathrm{M}\left(\mathbb{R}^{2}, S^{1}\right) \rtimes \mathrm{TOP}\left(\mathbb{R}^{2}\right)$. We work with

$$
1 \rightarrow \mathbb{Z}_{6} \rightarrow \Pi / K_{r} \rightarrow \mathbb{Z}^{2} \rightarrow 1
$$

A homomorphism

$$
\bar{\theta}: \Pi \rightarrow \Pi / K_{r} \rightarrow \mathrm{M}\left(\mathbb{R}^{2}, S^{1}\right) \rtimes \mathrm{TOP}\left(\mathbb{R}^{2}\right)
$$

sending $\bar{z}$ to $\omega^{-2}$ can be defined by a crossed homomorphism $\lambda: \Pi / K_{r} \rightarrow \mathrm{M}\left(\mathbb{R}^{2}, S^{1}\right)$. In fact,

$$
\lambda(x)\left(w_{1}, w_{2}\right)=1, \quad \lambda(y)\left(w_{1}, w_{2}\right)=\omega^{4 w_{1}}, \quad \text { and } \quad \lambda(\bar{z})\left(w_{1}, w_{2}\right)=\omega^{-2}
$$

gives rise to such a crossed homomorphism. By identifying $S^{1}$ to a subgroup of $G$, we obtain a representation $\Pi \rightarrow \Pi / K_{r} \rightarrow \mathrm{M}\left(\mathbb{R}^{2}, G\right) \rtimes \mathrm{TOP}\left(\mathbb{R}^{2}\right)$. In fact, setting up

$$
x \mapsto\left(1, t_{1}\right), \quad y \mapsto\left(\lambda(y), t_{2}\right), \quad z \mapsto\left(\omega^{-2}, 1\right)
$$


(so that $\lambda(x)=1$ and $\lambda(z)=\omega^{-2}$ ), one can get $\lambda(y)\left(w_{1}, w_{2}\right)=\omega^{4 w_{1}}$ from the relation $\bar{\theta}([x, y])=\bar{\theta}\left(\bar{z}^{2}\right)$.

Finally, combine these two maps to get $\Pi \rightarrow \ell(G) \times \mathrm{M}\left(\mathbb{R}^{2}, G\right) \rtimes \operatorname{TOP}\left(\mathbb{R}^{2}\right)$ :

$$
x \mapsto\left(1,1, t_{1}\right), \quad y \mapsto\left(1, \lambda, t_{2}\right), \quad z \mapsto\left(\omega^{3}, \omega^{-2}, 1\right) .
$$

The action of $\Pi$ on $G \times \mathbb{R}^{2}$ defined by this representation can be given explicitly as follows. Recall that $\omega=\mathcal{R}\left(\frac{1}{12}\right)$ and $(a, \lambda, \alpha) \cdot(v, w)=\left(a \cdot v \cdot \lambda(\alpha w)^{-1}, \alpha w\right)$ :

$$
\begin{aligned}
& x\left(v, w_{1}, w_{2}\right)=\left(v, \quad w_{1}+1, \quad w_{2}\right), \\
& y\left(v, w_{1}, w_{2}\right)=\left(v \omega^{-4 w_{1}}, \quad w_{1}, \quad w_{2}+1\right), \\
& z\left(v, w_{1}, w_{2}\right)=\left(\omega^{3} v \omega^{2}, \quad w_{1}, \quad w_{2}\right)
\end{aligned}
$$

for $\left(v, w_{1}, w_{2}\right) \in G \times \mathbb{R}^{2}$. Consequently, $\Pi \backslash\left(G \times \mathbb{R}^{2}\right)$ is a Seifert manifold modeled on $G \times \mathbb{R}^{2}$ with base a Riemannian flat torus $\mathbb{R}^{2} / \mathbb{Z}^{2}$.

From the presentation of the group $\Pi / K_{r}$, we see that $\mathcal{E}\left(\Pi / K_{r}\right)=1 \cdot(-2) \neq 0$ mod 6 , so that the condition $(\mathbf{C 2})$ is not satisfied. Therefore, the group $\Pi$ cannot be embedded into $\operatorname{Isom}\left(G \times \mathbb{R}^{2}\right)=\mathrm{SO}(4) \times E(2)$, and it cannot act as isometries on the product $G \times \mathbb{R}^{2}$.

The Seifert fibering is actually a fiber bundle over the 2-torus with fiber the lens space $L(12,5)$. So, no fiber is typical in this case. Since $\Pi$ is not a product $\mathbb{Z}_{12} \times Q$, the bundle is non-trivial. Moreover, the bundle contains an $S^{1}$-bundle over the same torus with euler class -8 . This is apparent from the diagram below, which describes the construction. In the diagram, Nil denotes the 3-dimensional Heisenberg group diffeomorphic (but not isometric) to $\mathbb{R} \times \mathbb{R}^{2}$, and $S^{1}$ denotes $\mathbb{R} / 12 \mathbb{Z}$.

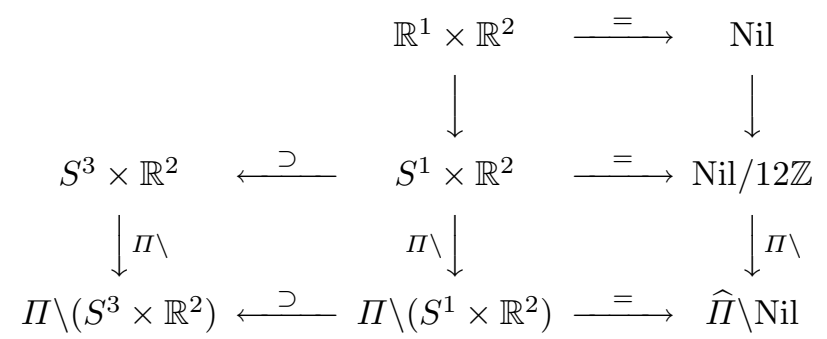

One needs to check only that the subset $S^{1} \times \mathbb{R}^{2}$ of $S^{3} \times \mathbb{R}^{2}$ is invariant under the action of $\Pi$. Furthermore, in the description of the action above, if $v \in S^{3}$ lies in $S^{1}$, the element $z \in \Pi$ acts as $z\left(v, w_{1}, w_{2}\right)=\left(v \omega^{5}, w_{1}, w_{2}\right)$, because $\omega^{3} v \omega^{2}=v \omega^{5}$ on $S^{1}$.

The case of $r=11$, so that $z \mapsto\left(\omega^{6}, \omega^{-5}\right)$, has more variations than the case we discussed above. This is because $\Pi / K_{\ell}=\mathbb{Z}_{2} \times \mathbb{Z}^{2}$. According to Theorem 4.7, we need to find a homomorphism $\Pi / K_{\ell} \rightarrow S^{3}$. Choose any homomorphism $\nu$ : $\mathbb{Z}^{2} \rightarrow S^{3}$. This together with the inclusion $\mathbb{Z}_{2} \hookrightarrow S^{3}$ gives rise to a homomorphism $\Pi / K_{\ell}=\mathbb{Z}_{2} \times \mathbb{Z}^{2} \rightarrow S^{3}$. For $\Pi / K_{r}$, the situation is the same as before.

An action of $\Pi$ on $G \times \mathbb{R}^{2}$ with trivial $\nu$ can be given explicitly as follows:

$$
\begin{aligned}
& x\left(v, w_{1}, w_{2}\right)=\left(v, \quad w_{1}+1, \quad w_{2}\right), \\
& y\left(v, w_{1}, w_{2}\right)=\left(v \omega^{8 w_{1}}, \quad w_{1}, \quad w_{2}+1\right), \\
& z\left(v, w_{1}, w_{2}\right)=\left(\omega^{6} v \omega^{5}, \quad w_{1}, \quad w_{2}\right)
\end{aligned}
$$


for $\left(v, w_{1}, w_{2}\right) \in G \times \mathbb{R}^{2}$. Consequently, $\Pi \backslash\left(G \times \mathbb{R}^{2}\right)$ is a Seifert manifold modeled on $G \times \mathbb{R}^{2}$ with base a Riemannian flat torus $\mathbb{R}^{2} / \mathbb{Z}^{2}$. Again, the Seifert fibering is an actual bundle over the torus, with fiber the lens space $L(12,11)$. This bundle is non-trivial since $\Pi$ is not a product. The bundle also contains an $S^{1}$-bundle over the same torus with euler class -8 . Also, it is not possible to embed $\Pi$ into $\operatorname{Isom}\left(S^{3} \times W\right)$ since condition $(\mathbf{C 2})$ is not satisfied for $\Pi / K_{r}$.

4.9. Example. Let $\widehat{Q}$ be a group with presentation

$$
\begin{gathered}
\widehat{Q}=\left\langle x_{1}, \cdots, x_{g}, y_{1}, \cdots, y_{g}, w_{1}, \cdots, w_{p}, z\right| z \text { central, } z^{m}=1, w_{j}{ }^{\alpha_{j}}=z^{-\beta_{j}}, \\
\left.\prod_{j=1}^{p} w_{j} \prod_{i=1}^{g}\left[x_{i}, y_{i}\right]=z^{b}\right\rangle
\end{gathered}
$$

with $(m, 6)=1$, and $\Pi=\mathbf{T}^{*} \times \widehat{Q}$. Then $\Delta=\mathbf{T}^{*} \times \mathbb{Z}_{m}$ is a spherical space-form group, and $Q=\Pi / \Delta$ has a presentation $(\mathbf{Q})$.

With $\Delta \hookrightarrow \ell(G) \times r(G)$ such that $\mathbf{T}^{*} \subset \ell(G)$ and $\mathbb{Z}_{m} \subset r(G)$, the condition (B) for $\Pi / K_{\ell}=\mathbf{T}^{*} \times Q$ is satisfied, so that there exists $\Pi \hookrightarrow \widetilde{\mathcal{U}}$ yielding a Seifert fiber space $\Pi \backslash(G \times W)$ with typical fiber $\mathbf{T}^{*} \backslash S^{3}$. If, furthermore, $\mathcal{E}(\widehat{Q})=0$, then $\Pi$ can be embed into $\operatorname{Isom}(G \times W)$.

On the other hand, with $\Delta \hookrightarrow \ell(G) \times r(G)$ such that $\mathbb{Z}_{m} \subset \ell(G)$ and $\mathbf{T}^{*} \subset r(G)$, in order for $\Pi$ to be embedded into $\operatorname{Isom}(G \times W)$, it is necessary and sufficient that the condition $(\mathbf{C 2})$ for $\Pi / K_{\ell}=\widehat{Q}$, which is $\mathcal{E}(\widehat{Q})=0$, is satisfied.

\section{Deformation of Seifert Structures}

Consider a commutative diagram of group homomorphisms with exact rows:

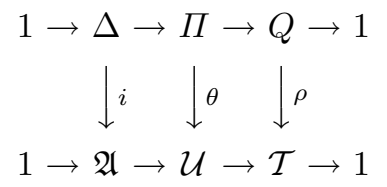

where $i$ is injective. With the homomorphisms $i$ and $\rho$ fixed, how many $\theta$ 's are there, keeping the diagram commutative? In the following, we identify $a \in \Delta$ with $i(a) \in \mathfrak{A}$, for brevity. We fix such a homomorphism $\theta_{0}$ once and for all and use the action of $\Pi$ on $\mathcal{U}$ gotten from $\Pi \stackrel{\theta_{0}}{\longrightarrow} \mathcal{U} \stackrel{\mu}{\longrightarrow} \operatorname{Aut}(\mathcal{U})$. Therefore, for $\alpha \in \Pi$,

$$
{ }^{\alpha} u=\theta_{0}(\alpha) \cdot u \cdot \theta_{0}(\alpha)^{-1}
$$

for any $u \in \mathcal{U}$.

Let $\theta: \Pi \rightarrow \mathcal{U}$ be such a homomorphism fitting the diagram. This $\theta$ will be expressed via the fixed $\theta_{0}$. Then $\theta$ must be of the form

$$
\theta(\alpha)=\lambda(\alpha) \theta_{0}(\alpha)
$$

for some map $\lambda: \Pi \rightarrow \mathfrak{A}$. It is easy to verify that $\lambda$ satisfies

$$
\begin{aligned}
\lambda(\alpha \beta) & =\lambda(\alpha) \cdot \theta_{0}(\alpha) \lambda(\beta) \theta_{0}(\alpha)^{-1} \\
& =\lambda(\alpha) \cdot{ }^{\alpha} \lambda(\beta) .
\end{aligned}
$$


Since $\left.\theta\right|_{\Delta}=i=\left.\theta_{0}\right|_{\Delta}$, we have $\lambda(a)=1$ for all $a \in \Delta$. For any $a \in \Delta$ and $\alpha \in \Pi$, $\lambda(\alpha a)=\lambda(\alpha)$. Moreover,

$$
\begin{aligned}
\lambda(\alpha) & =\lambda\left(\alpha \cdot\left(\alpha^{-1} a \alpha\right)\right)=\lambda(a \alpha)=\lambda(a) \cdot \theta_{0}(a) \lambda(\alpha) \theta_{0}(a)^{-1} \\
& =1 \cdot i(a) \lambda(\alpha) i(a)^{-1}=i(a) \lambda(\alpha) i(a)^{-1} .
\end{aligned}
$$

This shows $\lambda$ has values in the centralizer $C_{\mathfrak{A}}(\Delta)$, which depends only upon the image of $\alpha$ in $Q$. Furthermore, (5.0.2) shows that $\theta_{0}(\alpha) \lambda(\beta) \theta_{0}(\alpha)^{-1} \in C_{\mathfrak{A}}(\Delta)$. Therefore,

$$
\theta_{0}(\alpha) \lambda(\beta) \theta_{0}(\alpha)^{-1}=i(a) \theta_{0}(\alpha) \lambda(\beta) \theta_{0}(\alpha)^{-1} i(a)^{-1}=\theta_{0}(a \alpha) \lambda(\beta) \theta_{0}(a \alpha)^{-1} .
$$

Let $\mathfrak{C}$ be the subgroup of $\mathcal{U}$ defined as:

$$
\mathfrak{C}=\{c \in \mathcal{U}: \mu(c) \text { induces the identity on } i(\Delta) \text { and } \rho(Q)\} .
$$

Then

$$
1 \rightarrow C_{\mathfrak{A}}(\Delta) \rightarrow \mathfrak{C} \rightarrow C_{\mathcal{T}}(\rho(Q)) \rightarrow 1
$$

is exact.

Let $c \in \mathfrak{C}$ and $\mu\left(c^{-1}\right)\left(\theta_{0}(\alpha)\right)=\lambda(\alpha) \theta_{0}(\alpha)$. Then $\theta_{0}(\alpha) c \theta_{0}(\alpha)^{-1}=c \lambda(\alpha)$. Since $i(a)$ commutes with $c, i(a)$ commutes with $c \lambda(\alpha)$. Using (5.0.3), we get

$$
\begin{aligned}
\theta_{0}(\alpha) c \theta_{0}(\alpha)^{-1} & =c \lambda(\alpha)=i(a) c \lambda(\alpha) i(a)^{-1} \\
& =i(a) \theta_{0}(\alpha) c \theta_{0}(\alpha)^{-1} i(a)^{-1}=\theta_{0}(a \alpha) c \theta_{0}(a \alpha)^{-1} .
\end{aligned}
$$

By (5.0.3) and (5.0.4), the actions of $\Pi$ induced by $\mu \circ \theta_{0}$ on $\mathfrak{C}$ and on $C_{\mathfrak{A}}(\Delta)$ factor through $Q$. With these homomorphisms $Q \rightarrow \operatorname{Aut}(\mathfrak{C})$ and $Q \rightarrow \operatorname{Aut}\left(C_{\mathfrak{A}}(\Delta)\right)$, we may consider $Z^{1}\left(Q ; C_{\mathfrak{A}}(\Delta)\right)$ and $B^{1}(Q ; \mathfrak{C})$. The equality (5.0.2) shows that $\lambda \in Z^{1}\left(Q ; C_{\mathfrak{A}}(\Delta)\right)$. Therefore,

Lemma 5.1. $Z^{1}\left(Q ; C_{\mathfrak{A}}(\Delta)\right)$ represents the set of all homomorphisms $\theta$ 's fitting the commutative diagram (5.0.1). In fact, $\lambda \longleftrightarrow \theta=\lambda \cdot \theta_{0}$ gives the one-one correspondence

$$
\begin{aligned}
Z^{1}\left(Q ; C_{\mathfrak{A}}(\Delta)\right) & =\left\{\lambda: Q \rightarrow C_{\mathfrak{A}}(\Delta) \mid \lambda(\alpha \beta)=\lambda(\alpha) \cdot{ }^{\alpha} \lambda(\beta)\right\} \\
& \cong \\
\stackrel{\cong}{\Leftrightarrow} & \{\theta: \Pi \rightarrow \mathcal{U} \text { inducing } i \text { and } \rho \text { on } \Delta \text { and on } Q\} .
\end{aligned}
$$

The group $\mathfrak{C}$ acts on $Z^{1}\left(Q ; C_{\mathfrak{A}}(\Delta)\right)$ as follows: Let $c \in \mathfrak{C}, \lambda \in Z^{1}\left(Q ; C_{\mathfrak{A}}(\Delta)\right)$. Then ${ }^{c} \lambda$ is given by

$$
\begin{aligned}
\left({ }^{c} \lambda\right)(\alpha) & =c \cdot \lambda(\alpha) \cdot{ }^{\alpha} c^{-1} \\
& =c \cdot \lambda(\alpha) \cdot \theta_{0}(\alpha) \cdot c^{-1} \cdot \theta_{0}(\alpha)^{-1} .
\end{aligned}
$$

It is easy to see that $\left({ }^{c} \lambda\right)(\alpha) \in C_{\mathfrak{A}}(\Delta)$, and ${ }^{c} \lambda$ satisfies the cocycle condition. Then the subgroup $\left.C_{\mathfrak{A}}(\Delta)\right) \subset \mathfrak{C}$ acts on $Z^{1}\left(Q ; C_{\mathfrak{A}}(\Delta)\right.$ as well.

After the abelian case, we denote the orbit spaces of these actions by

$$
H^{1}\left(Q ; C_{\mathfrak{A}}(\Delta)\right) \equiv \frac{Z^{1}\left(Q ; C_{\mathfrak{A}}(\Delta)\right)}{B^{1}\left(Q ; C_{\mathfrak{A}}(\Delta)\right)} \quad \text { and } \quad \frac{Z^{1}\left(Q ; C_{\mathfrak{A}}(\Delta)\right)}{B^{1}(Q ; \mathfrak{C})}
$$

Lemma 5.2. $\theta_{1}=\lambda_{1} \cdot \theta_{0}$ and $\theta_{2}=\lambda_{2} \cdot \theta_{0}$ are conjugate by an element of $\mathfrak{A}$ if and only if $\lambda_{1}$ and $\lambda_{2}$ belong to the same orbit of the $C_{\mathfrak{A}}(\Delta)$-action. 
Proof. Suppose there exists $a \in \mathfrak{A}$ so that

$$
\theta_{2}(\alpha)=a \theta_{1}(\alpha) a^{-1}
$$

for all $\alpha \in \Pi$. Since $\theta_{1}=\theta_{0}=\theta_{2}$ on $\Delta, \mu(a)$ induces the identity on $i(\Delta)$, and hence, $a$ must be in $C_{\mathfrak{A}}(\Delta)$. Then $\lambda_{2}(\alpha) \theta_{0}(\alpha)=a \lambda_{1}(\alpha) \theta_{0}(\alpha) a^{-1}$ yields

$$
\lambda_{2}(\alpha)=a \cdot \lambda_{1}(\alpha) \cdot{ }^{\alpha} a^{-1} .
$$

Thus, $\lambda_{1}$ and $\lambda_{2}$ belong to the same orbit of the $C_{\mathfrak{A}}(\Delta)$-action. Conversely, the above equality easily implies $\theta_{2}(\alpha)=a \theta_{1}(\alpha) a^{-1}$.

Lemma 5.3. $\theta_{1}=\lambda_{1} \cdot \theta_{0}$ and $\theta_{2}=\lambda_{2} \cdot \theta_{0}$ are conjugate by an element of $\mathcal{U}$ if and only if $\lambda_{1}$ and $\lambda_{2}$ belong to the same orbit of the $\mathfrak{C}$-action.

Proof. Suppose there exists $c \in \mathcal{U}$ so that

$$
\theta_{2}(\alpha)=c \theta_{1}(\alpha) c^{-1}
$$

for all $\alpha \in \Pi$. Since $\theta_{1}, \theta_{2}$ induce $i$ on $\Delta$, and $\rho$ on $Q, c$ must be in $\mathfrak{C}$. Also, $\lambda_{2} \theta_{0}(\alpha)=c \lambda_{1} \theta_{0}(\alpha) c^{-1}$ yields

$$
\lambda_{2}(\alpha)=c \cdot \lambda_{1}(\alpha) \cdot{ }^{\alpha} c^{-1} .
$$

Thus, $\lambda_{1}$ and $\lambda_{2}$ belong to the same orbit of the $\mathfrak{C}$-action. Conversely, the above equality easily implies $\theta_{2}(\alpha)=c \theta_{1}(\alpha) c^{-1}$.

Theorem 5.4. The set of all homomorphisms $\theta$ fitting the commutative (5.0.1) diagram with the fixed $i$ and $\rho$ are classified as follows:

$$
\begin{aligned}
& H^{1}\left(Q ; C_{\mathfrak{A}}(\Delta)\right) \equiv \frac{Z^{1}\left(Q ; C_{\mathfrak{A}}(\Delta)\right)}{B^{1}\left(Q ; C_{\mathfrak{A}}(\Delta)\right)} \stackrel{\cong}{\longleftrightarrow}\{\theta: \Pi \rightarrow \mathcal{U} \text { inducing } i \text { and } \rho \text { on } \Delta \text { and on } Q\} \\
& \text { up to conjugation by elements of } \mathfrak{A} \\
& \frac{Z^{1}\left(Q ; C_{\mathfrak{A}}(\Delta)\right)}{B^{1}(Q ; \mathfrak{C})} \stackrel{\cong}{\longleftrightarrow}\{\theta: \Pi \rightarrow \mathcal{U} \text { inducing } i \text { and } \rho \text { on } \Delta \text { and on } Q\} \\
& \text { up to conjugation by elements of } \mathcal{U} \text {. }
\end{aligned}
$$

Conjugation of $\theta_{0}$ by any element $u$ of $\mathcal{U}$ gives rise to a new embedding $\theta: \Pi \rightarrow \mathcal{U}$ which maps $\Delta$ necessarily into $\mathrm{M}(W, G) \rtimes \operatorname{Inn}(G)$, since $\mathrm{M}(W, G) \rtimes \operatorname{Inn}(G)$ is normal in $\mathcal{U}$. Then this induces a Seifert automorphism between the Seifert fiber spaces $\theta_{0}(\Pi) \backslash(G \times W)$ and $\theta(\Pi) \backslash(G \times W)$. If $u \in \mathfrak{C}$, then the Seifert automorphism is called a strict Seifert automorphism. When $u \in \mathrm{M}(W, G) \rtimes \operatorname{Inn}(G)$, the Seifert automorphism is a strict Seifert automorphism moving only along the fibers. Therefore, $H^{1}\left(Q ; C_{\mathfrak{A}}(\Delta)\right)$ classifies all strict Seifert automorphism moving only along the fibers, while $\frac{Z^{1}\left(Q ; C_{\mathfrak{A}}(\Delta)\right)}{B^{1}(Q ; \mathcal{C})}$ classifies all strict Seifert automorphism. General Seifert automorphisms come from the quotient space of the discrete faithful representations of $\Pi$ into $\mathcal{U}$ by the conjugations from elements of $\mathcal{U}$.

For our application with $\mathfrak{A}=\ell(G) \times \mathrm{M}(W, G)$, we have $C_{\ell(G) \times \mathrm{M}(W, G)}(\Delta)=$ $\left.C_{\ell(G)}\left(\Delta_{\ell}\right) \times \mathrm{M}\left(W, C_{G}\left(\Delta_{r}\right)\right)\right)$. Therefore,

$$
H^{1}\left(Q ; C_{\mathfrak{A}}(\Delta)\right)=H^{1}\left(Q ; C_{\ell(G)}\left(\Delta_{\ell}\right)\right) \oplus H^{1}\left(Q ; \mathrm{M}\left(W, C_{G}\left(\Delta_{r}\right)\right)\right) .
$$

Note that when $Q \rightarrow \operatorname{Aut}\left(C_{\ell(G)}\left(\Delta_{\ell}\right)\right)$ is trivial,

$$
H^{1}\left(Q ; C_{\ell(G)}\left(\Delta_{\ell}\right)\right)=\operatorname{Hom}\left(Q ; C_{\ell(G)}\left(\Delta_{\ell}\right)\right) .
$$


Example 5.5. We continue with Example 4.8. For the first case with $z \mapsto$ $\left(\omega^{3}, \omega^{-2}\right)$, we have $\Delta_{\ell}=\mathbb{Z}_{4}$ and $\Delta_{r}=\mathbb{Z}_{6}$, so that $C_{\ell(G)}\left(\Delta_{\ell}\right)=\mathrm{SO}(2)$ and $\mathrm{M}\left(W, C_{G}\left(\Delta_{r}\right)\right)=\mathrm{M}(W, \mathrm{SO}(2))$. Clearly, $H^{1}(Q ; \mathrm{SO}(2))=\operatorname{Hom}(Q, \mathrm{SO}(2))=S^{1} \times$ $S^{1}=T^{2}$. To calculate $H^{1}\left(Q ; \mathrm{M}\left(W, C_{G}\left(\Delta_{r}\right)\right)\right)$, consider the short exact sequence of $Q$-modules $1 \rightarrow \mathbb{Z} \rightarrow \mathbb{R} \rightarrow \mathrm{SO}(2) \rightarrow 1$. It yields an exact sequence

$$
H^{1}(Q ; \mathrm{M}(W, \mathbb{R})) \rightarrow H^{1}(Q ; \mathrm{M}(W, \mathrm{SO}(2))) \rightarrow H^{2}(Q ; \mathbb{Z}) \rightarrow H^{2}(Q ; \mathrm{M}(W, \mathbb{R})) .
$$

Since $H^{i}(Q ; \mathrm{M}(W, \mathbb{R}))=0$ for $i>0[2]$, we get $H^{1}(Q ; \mathrm{M}(W, \mathrm{SO}(2))) \cong H^{2}(Q ; \mathbb{Z})=$ $\mathbb{Z}$. A cocycle $\lambda_{p}$ corresponding to $p \in \mathbb{Z}=H^{1}(Q ; \mathrm{M}(W, \mathrm{SO}(2)))$ can be described as

$$
\lambda_{p}\left(t_{1}\right)\left(w_{1}, w_{2}\right)=e^{-2 \pi i \cdot p w_{2}}, \quad \lambda_{p}\left(t_{2}\right)\left(w_{1}, w_{2}\right)=1 .
$$

Then $\delta \lambda_{p} \in H^{2}(Q ; \mathbb{Z})$ is given by $\delta \lambda_{p}\left(t_{1}, t_{2}\right)=0$ and $\delta \lambda_{p}\left(t_{2}, t_{1}\right)=z^{p}$. Consequently,

$$
H^{1}\left(Q ; C_{\ell(G) \times \mathrm{M}(W, G)}(\Delta)\right)=T^{2} \times \mathbb{Z}
$$

With $\tilde{\mathcal{U}}=\ell(G) \times[\mathrm{M}(W, G) \rtimes \operatorname{Isom}(W)]$, conjugation by elements of $\tilde{\mathcal{U}}$ does not contribute anything new. In other words,

$$
Z^{1}\left(Q ; C_{\mathfrak{A}}(\Delta)\right) / B^{1}\left(Q ; C_{\mathfrak{A}}(\Delta)\right)=Z^{1}\left(Q ; C_{\mathfrak{A}}(\Delta)\right) / B^{1}(Q ; \mathfrak{C})
$$

The centralizer of $Q$ in $\operatorname{Isom}\left(\mathbb{R}^{2}\right)$ is the pure translations $\mathbb{R}^{2}$. For $t_{b}=\left(b_{1}, b_{2}\right) \in$ $\mathbb{R}^{2}, \mu\left(t_{b}\right)$ can be achieved by $\mu(\eta)$, where $\eta \in B^{1}(Q ; \mathrm{M}(W, \mathrm{SO}(2)))$ is defined by $\eta\left(w_{1}, w_{2}\right)=\omega^{4 b_{1}}$. Thus,

$$
H^{1}\left(Q ; C_{\mathfrak{A}}(\Delta)\right) \equiv \frac{Z^{1}\left(Q ; C_{\mathfrak{A}}(\Delta)\right)}{B^{1}\left(Q ; C_{\mathfrak{A}}(\Delta)\right)} \cong \frac{Z^{1}\left(Q ; C_{\mathfrak{A}}(\Delta)\right)}{B^{1}(Q ; \mathfrak{C})}
$$

Corresponding to $((a, b), p) \in T^{2} \times \mathbb{Z}=H^{1}\left(Q ; C_{\ell(G) \times \mathrm{M}(W, G)}(\Delta)\right)$, the action of $\Pi$ on $G \times \mathbb{R}^{2}$ is given by:

$$
\begin{array}{lll}
x\left(v, w_{1}, w_{2}\right)=\left(\begin{array}{lll}
a v e^{2 \pi i \cdot p w_{2}}, & w_{1}+1, & w_{2}
\end{array}\right), \\
y\left(v, w_{1}, w_{2}\right)=\left(\begin{array}{lll}
b v \omega^{-4 w_{1}}, & w_{1}, & w_{2}+1
\end{array}\right), \\
z\left(v, w_{1}, w_{2}\right)=\left(\begin{array}{lll}
\omega^{3} v \omega^{2}, & w_{1}, & w_{2}
\end{array}\right)
\end{array}
$$

for $\left(v, w_{1}, w_{2}\right) \in G \times \mathbb{R}^{2}$.

In the second case with $z \mapsto\left(\omega^{6}, \omega^{-5}\right)$, we have $\Delta_{\ell}=\mathbb{Z}_{2}=\mathcal{Z}\left(S^{3}\right)$ and $\Delta_{r}=\mathbb{Z}_{12}$, so that $C_{\ell(G)}\left(\Delta_{\ell}\right)=S^{3}$ and $\mathrm{M}\left(W, C_{G}\left(\Delta_{r}\right)\right)=\mathrm{M}(W, \mathrm{SO}(2))$. Now, $H^{1}\left(Q ; S^{3}\right)=$ $\operatorname{Hom}\left(Q, S^{3}\right)$. Therefore,

$$
H^{1}\left(Q ; C_{\ell(G) \times \mathrm{M}(W, G)}(\Delta)\right)=\operatorname{Hom}\left(Q, S^{3}\right) \times \mathbb{Z} .
$$

Similarly to the previous case, with $\widetilde{\mathcal{U}}=\ell(G) \times[\mathrm{M}(W, G) \rtimes \operatorname{Isom}(W)]$, conjugation by elements of $\widetilde{\mathcal{U}}$ does not contribute anything new. The centralizer of $Q$ in $\operatorname{Isom}\left(\mathbb{R}^{2}\right)$ is the pure translations $\mathbb{R}^{2}$. For $b=\left(b_{1}, b_{2}\right) \in \mathbb{R}^{2}, \mu\left(t_{b}\right)$ can be achieved by $\mu(\eta)$, where $\eta\left(w_{1}, w_{2}\right)=\omega^{-8 b_{1}} \in B^{1}(Q ; \mathrm{M}(W, \mathrm{SO}(2)))$.

Corresponding to $((a, b), p) \in \operatorname{Hom}\left(\mathbb{Z}^{2}, S^{3}\right) \times \mathbb{Z}=H^{1}\left(Q ; C_{\ell(G) \times \mathrm{M}(W, G)}(\Delta)\right)$, the action of $\Pi$ on $G \times \mathbb{R}^{2}$ is given by:

$$
\begin{array}{lll}
x\left(v, w_{1}, w_{2}\right)=\left(\begin{array}{lll}
a v e^{2 \pi i \cdot p w_{2}}, & w_{1}+1, & w_{2}
\end{array}\right), \\
y\left(v, w_{1}, w_{2}\right)=\left(\begin{array}{lll}
b v \omega^{8 w_{1}}, & w_{1}, & w_{2}+1
\end{array}\right), \\
z\left(v, w_{1}, w_{2}\right)=\left(\begin{array}{lll}
\omega^{3} v \omega^{2}, & w_{1}, & w_{2}
\end{array}\right)
\end{array}
$$

for $\left(v, w_{1}, w_{2}\right) \in G \times \mathbb{R}^{2}$. 
We claim that, in both cases, the Seifert fiber spaces coming from the representations corresponding to $H^{1}\left(Q ; C_{\ell(G)}(\Delta)\right)=T^{2}$ or $\operatorname{Hom}\left(\mathbb{Z}^{2}, S^{3}\right)$ are $h$-cobordant to $\theta_{0}(\Pi) \backslash\left(S^{3} \times \mathbb{R}^{2}\right)$. With $Q=\mathbb{Z}^{2}, \operatorname{Hom}\left(Q, S^{3}\right)$ can be calculated as follows: Let $t_{1}, t_{2}$ be two generators of $\mathbb{Z}^{2}$. Map $t_{1} \mapsto \lambda\left(t_{1}\right) \in S^{3}$ anywhere. Suppose $\lambda\left(t_{1}\right) \notin \mathcal{Z}\left(S^{3}\right)$. That is, the order of $\lambda\left(t_{1}\right)$ is greater than 2 . Then $\lambda\left(t_{2}\right)$ must be in the centralizer of $\lambda\left(t_{1}\right)$, which is the unique circle subgroup containing $\lambda\left(t_{1}\right)$. If $\lambda\left(t_{1}\right) \in \mathcal{Z}\left(S^{3}\right)$, then $\lambda\left(t_{2}\right)$ may be arbitrary. Thus, $\operatorname{Hom}\left(Q, S^{3}\right)$ is an $\mathrm{SO}(2)$-bundle over $S^{3}$ whose fibers at the two points in $\mathcal{Z}\left(S^{3}\right)$ are replaced by $S^{3}$. What is important is the fact that $\operatorname{Hom}(Q, \mathrm{SO}(2))$ and $\operatorname{Hom}\left(Q, S^{3}\right)$ are path-connected. Since $H^{1}\left(Q ; C_{\ell(G)}(\Delta)\right)$ is path-connected, one can find a 1-parameter family of homomorphisms $\nu_{t}: \mathbb{Z}^{2} \rightarrow$ $C_{\ell(G) \times \mathrm{M}(W, G)}(\Delta)$ starting at $\nu=1$ and ending at a new $\nu^{\prime}$. This 1 -parameter family of homomorphisms $\nu_{t}, 0 \leq t \leq 1$, gives rise to a 1-parameter family of Seifert fiber spaces $\theta_{t}(\Pi) \backslash\left(S^{3} \times \mathbb{R}^{2}\right)$ Consequently, the two spaces $\theta_{0}(\Pi) \backslash\left(S^{3} \times \mathbb{R}^{2}\right)$ and $\theta_{1}(\Pi) \backslash\left(S^{3} \times \mathbb{R}^{2}\right)$ are smoothly $h-$ cobordant. We don't know if the same holds for representations corresponding to $H^{1}(Q ; \mathrm{M}(W, \mathrm{SO}(2)))=\mathbb{Z}$.

Remark 5.6. We have exhibited different embeddings of $\Pi$ in $\widetilde{\mathcal{U}}$ for for $r=5$ and 11 . Moreover, as actions on $S^{3} \times \mathbb{R}^{2}$, these groups are not conjugate in $\widetilde{\mathcal{U}}$. (Because the typical fibers of the resulting Seifert 5 -manifolds would have to be homeomorphic since $\ell(G)$ is normalized by $\widetilde{\mathcal{U}}$. The typical fibers are $L(2,1)$ and $S^{3}$, respectively). As lens space bundles over the torus, the fibers of the 5-manifolds are obviously different. It is not clear to us whether or not these spaces are of the same homotopy types.

\section{REFERENCES}

[1] G. E. Bredon, Introduction to compact transformation groups, Academic Press, Inc., Orlando, FL, 1972. MR 54:1265

[2] P. E. Conner and F. Raymond, Actions of compact Lie groups on aspherical manifolds, (1969), Topology of Manifolds, edited by J.C. Cantrell and C.H. Edwards, Markham, Chicago, 227-264. MR 42:6839

[3] P. E. Conner and F. Raymond, Holomorphic Seifert fiberings, Proc. 2nd Conference on Transformations groups, Part II. Springer-Verlag, Lecture Notes in Math. vol. 299, (1971), 124-204. MR 58:28698

[4] P. E. Conner and F. Raymond, Deforming Homotopy Equivalences to Homeomorphisms in Aspherical Manifolds, Bull. Amer. Math. Soc. 83 (1977), 36-85. MR 57:7629

[5] P. Igodt and K. B. Lee, Applications of group cohomology to space constructions, Trans. Amer. Math. Soc. 304 (1987), 69-82. MR 88j:57045

[6] M. Jankins and W. Neumann, Lectures on Seifert manifolds, Brandeis University, Waltham, Mass., 1983. MR 85j:57015

[7] R. Kulkarni and F. Raymond, 3-dimensional Lorentz space-forms and Seifert fiber spaces, J. Differential Geom. 21 (1985), 231-268. MR 87h:53092

[8] K. B. Lee, Infra-solvmanifolds of type (R), Quart. J. Math. Oxford (2) 46 (1995), 185-195

[9] K. B. Lee and F. Raymond, The role of Seifert fiber spaces in transformation groups, Contemporary Math., A. M. S. 36 (1985), 367-425. MR 86j:57015

[10] K. B. Lee and F. Raymond, Seifert manifolds modeled on principal bundles, Transf. Groups (Osaka,1987); Springer-Verlag, Lecture Notes in Math. vol. 1375, (1989), 207-215. MR 90f: 57041

[11] S. S. MacLane, Homology, Springer-Verlag, 1963. MR 28:122

[12] J. Milnor, Groups which act on $S^{n}$ without fixed points, American J. Math. 79 (1957), 623630. MR 19:761d

[13] P. Orlik, Seifert Manifolds, Springer-Verlag, Lecture Notes in Math., vol. 291, 1972. MR 54:14590 
[14] M. M. Postnikov, Three-dimensional spherical forms, Trudy Mat. Inst. Steklov. 196 (1991), 114-146; Proc. Steklov Inst. Math. 1992, no. 4 (196), 129-161. MR 92j:53016 (Russian original)

[15] P. Scott, The geometries of 3-manifolds, Bull. London Math. Soc. 15 (1983), 401-487. MR 84m:57009

[16] F. Raymond and A. Vasquez, 3-manifolds whose universal coverings are Lie groups, Topology and Appl. 12 (1981), 161-179. MR 82i:57011

[17] F. Raymond and D. Wigner, Construction of aspherical manifolds, Geometric Appl. of Homotopy Theory I, Springer-Verlag, Lecture Notes in Math. vol. 657 (1978), 408-422. MR 80d:57003

[18] J. A. Wolf, Spaces of constant curvature, 4th ed., Publish or Perish, Inc., Berkeley, CA, 1977. MR 49:7958 (3rd ed.)

Department of Mathematics, Sogang University, Seoul 121-742, Korea

E-mail address: jlee@ccs.sogang.ac.kr

Department of Mathematics, University of Oklahoma, Norman, Oklahoma 73019

E-mail address: kblee@.math.uoknor.edu

Department of Mathematics, University of Michigan, Ann Arbor, Michigan 48109

E-mail address: fraymond@math.1sa.umich.edu 